\title{
Enabling Situation Awareness Under High Levels of Automation: Results From An Experimental Study
}

Katya Le Blanc

Zachary Spielman

Gordon Bower

Johanna Oxstrand

Aaron Bly

July 2015

The INL is a

U.S. Department of Energy

National Laboratory

operated by

Battelle Energy Alliance

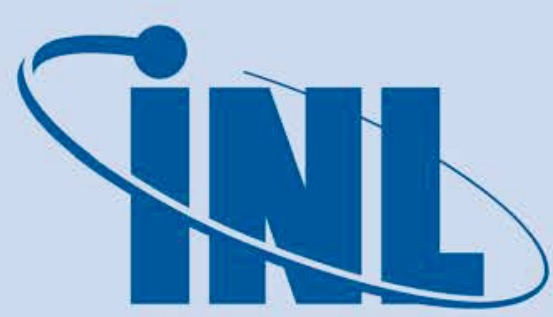

Idaho National Laboratory 


\section{DISCLAIMER}

This information was prepared as an account of work sponsored by an agency of the U.S. Government. Neither the U.S. Government nor any agency thereof, nor any of their employees, makes any warranty, expressed or implied, or assumes any legal liability or responsibility for the accuracy, completeness, or usefulness, of any information, apparatus, product, or process disclosed, or represents that its use would not infringe privately owned rights. References herein to any specific commercial product, process, or service by trade name, trade mark, manufacturer, or otherwise, does not necessarily constitute or imply its endorsement, recommendation, or favoring by the U.S. Government or any agency thereof. The views and opinions of authors expressed herein do not necessarily state or reflect those of the U.S. Government or any agency thereof. 
INL/EXT-15-35791

Revision 1

\title{
Enabling Situation Awareness Under High Levels of Automation: Results From An Experimental Study
}

\author{
Katya Le Blanc \\ Zachary Spielman \\ Gordon Bower \\ Johanna Oxstrand \\ Aaron Bly
}

July 2015

Idaho National Laboratory
INL ART TDO Program
Idaho Falls, Idaho 83415

http://www.inl.gov

Prepared for the

U.S. Department of Energy

Office of Nuclear Energy

Under DOE Idaho Operations Office

Contract DE-AC07-05ID14517 



\title{
INL ART TDO Program
}

\section{Enabling Situation Awareness Under High Levels of Automation: Results From An Experimental Study}

\author{
INL/EXT-15-35791 \\ Revision 0
}

July 2015

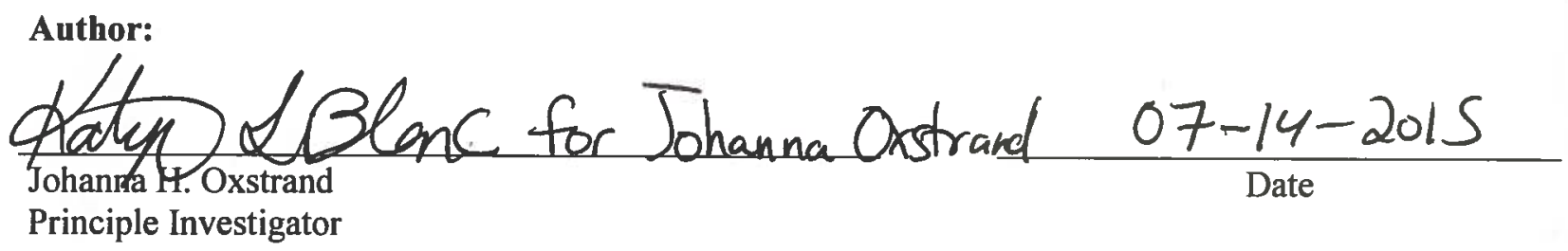

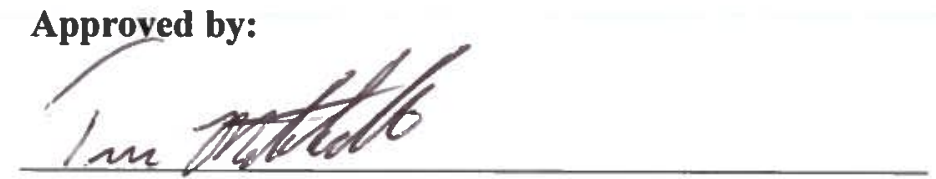

Travis R. Mitchell

INL ART TDO Relationship Manager

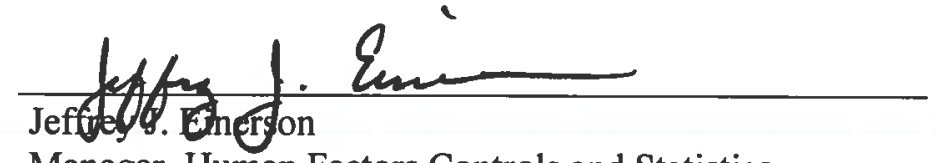

Manager, Human Factors Controls and Statistics

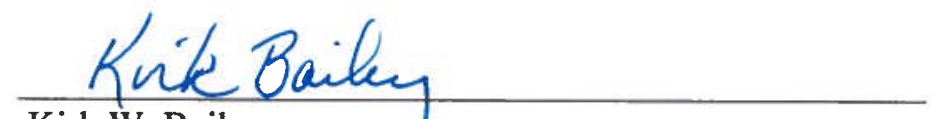

Kirk W. Bailey

INL ART TDO Quality Assurance

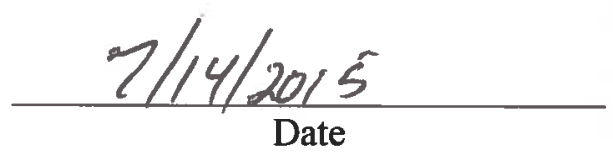

$\frac{7-14-2015}{\text { Date }}$

$\frac{7 \cdot 14 \cdot 2015}{\text { Date }}$ 



\section{ACKNOWLEDGEMENTS}

The research team would like to acknowledge Heather Medema and David Powers, Idaho National Laboratory, for their support in executing the 2015

experimental study. The team would also like to thank all who participated in the study. 


\section{SUMMARY}

Automation poses a great opportunity to increase both system and human performance. However, it is important to pay attention to the design of the collaboration between the human operator and the automated systems. If not designed correctly, the overall performance may be severely reduced. Previous research indicates that an intermediate level of automation might be the solution that minimizes the tradeoffs between manual and automated actions. However, there are still many remaining unanswered questions related to impacts on situation awareness (SA) and workload during different levels of automation (LOAs). Some of the documented issues of automation include reduced SA, complacency, and over reliance on the system automation.

The Advanced Reactor Technologies Human Automation Collaboration Research Project has conducted two experimental studies investigating the effect of different LOAs on system and human performance. The results from the first experimental study were published in Oxstrand and Le Blanc (2014) and Le Blanc and Oxstrand (2015) and are summarized in this report. The second experimental study was conducted by Idaho National Laboratory researchers. This report focuses on the second experimental study and details the design of the study and results.

The second experimental study aimed to investigate the effects automation has on overall human-system performance. Researchers employed a simplified process control simulation using a nested experimental design to evaluate performance differences between four LOAs (manual, intermediate, adaptable, and automatic) with varying faults and conditional changes occurring during each scenario. The effects of automation were measured by participants' ability to detect and respond to failing automation and conditional changes using a variety of metrics assessing SA, system performance, and workload. The intent of this study is to determine the optimum conditions for human automation collaboration.

The results of the present study indicate that more automation led to better system performance under normal operating conditions, and poorer SA and system performance under fault conditions. The results for the two middle LOAs are not as straightforward. Consistent with the hypothesis, system performance using adaptable automation exceeded system performance when using the intermediate LOA. However, contrary to the hypotheses, SA and fault performance were not superior using the adaptable LOA than using the intermediate LOA. The results of this study indicate that although adaptable automation fulfills half if its promise (i.e., it produces system performance closer to fully automatic than intermediate LOAs), it falls short of also enhancing SA and fault performance. This study demonstrates the fundamental tradeoff associated with high LOAs: automation enhances performance during normal conditions, but increases failure (sometimes catastrophically) of the humansystem under the condition of automation failure. In contrast, although manual performance is inferior to automatic performance under normal conditions, it is more stable, and is superior to automatic performance under automation failure conditions. Intermediate automation provided similarly good fault management performance, but did not enhance system performance as well as adaptable automation under normal conditions did. 
Unfortunately, the present study did not demonstrate that adaptable automation is an effective approach to avoiding that fundamental tradeoff and achieving an optimal combination of system performance, SA and fault management performance. Further research is needed to identify an approach to human automation collaboration that will enable higher LOAs without succumbing to the fundamental tradeoffs of automation. 


\section{CONTENTS}

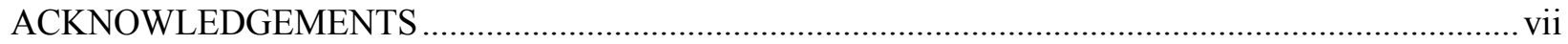

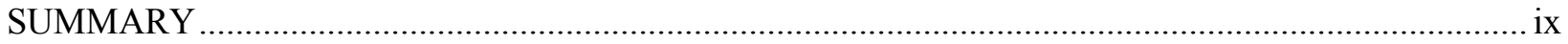

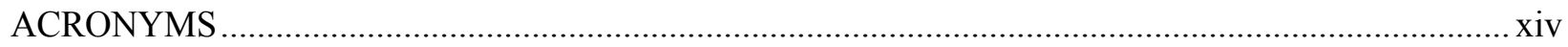

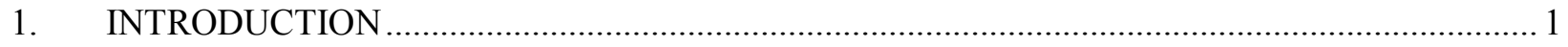

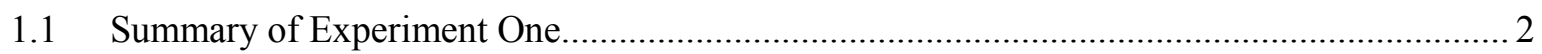

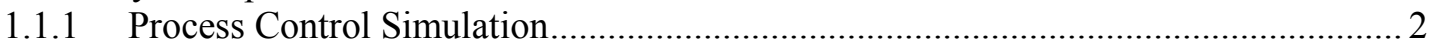

1.1.2 Summary of Experiment One Methods and Results .............................................. 3

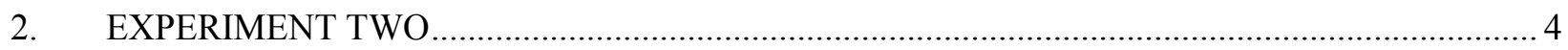

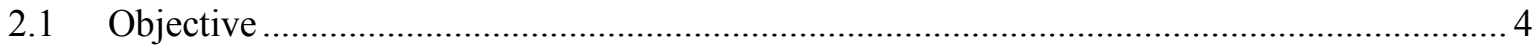

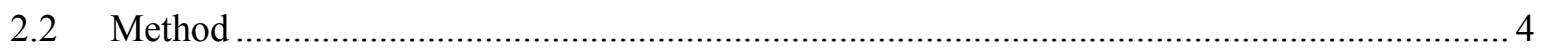

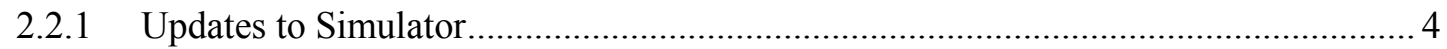

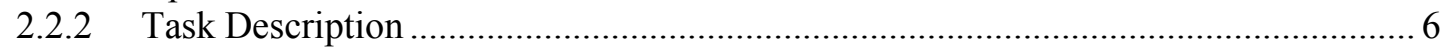

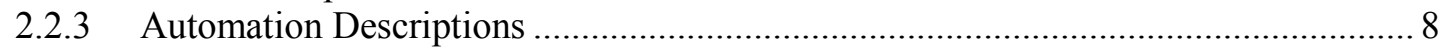

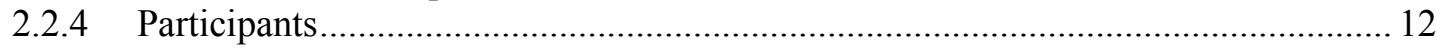

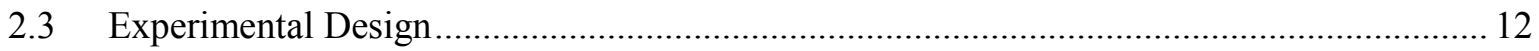

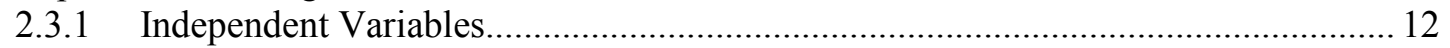

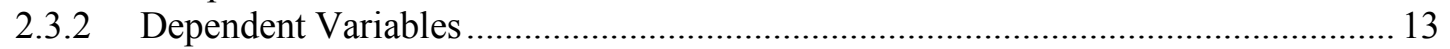

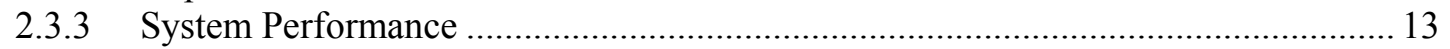

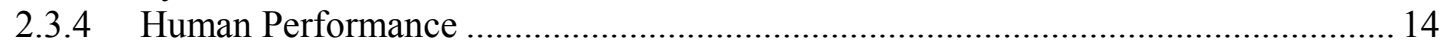

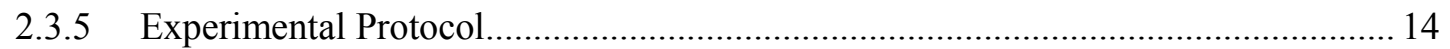

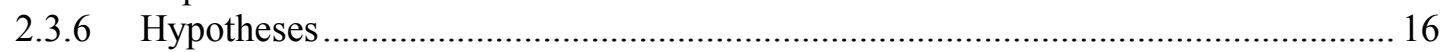

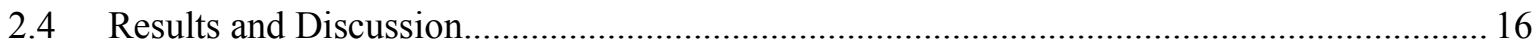

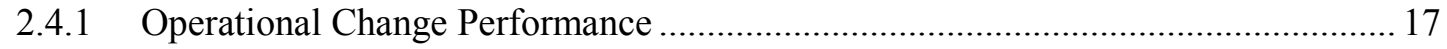

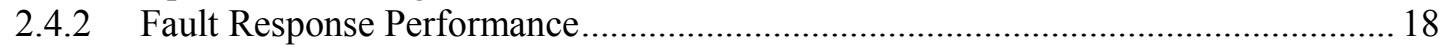

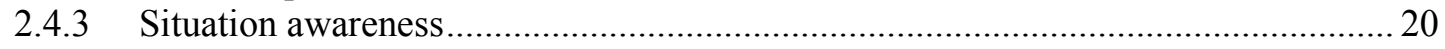

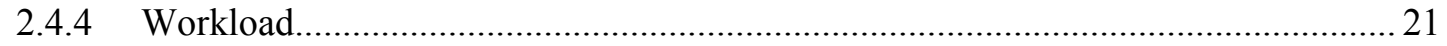

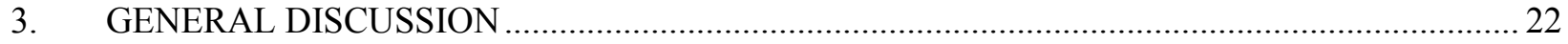

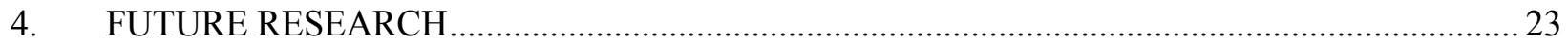

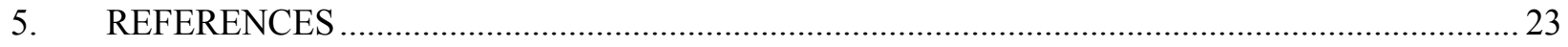

FIGURES

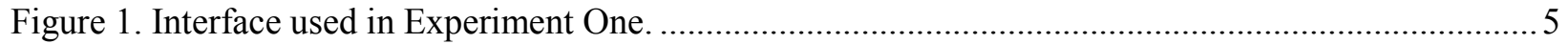

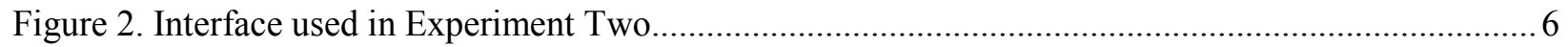

Figure 3. The control tank configuration of SMR Chemical............................................................... 7

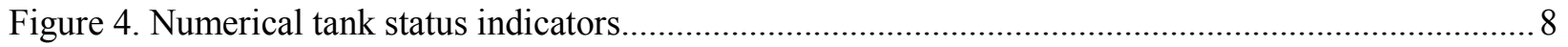

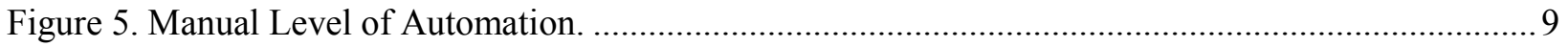

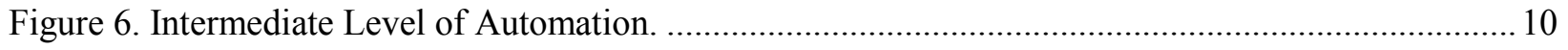




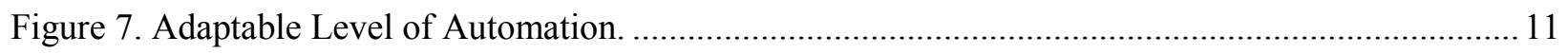

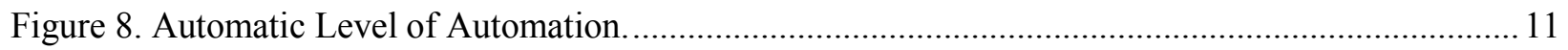

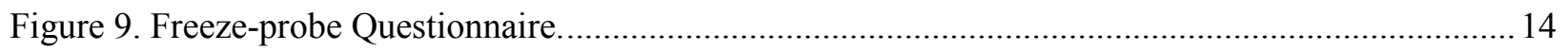

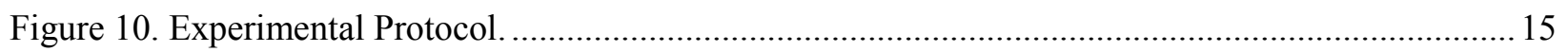

Figure 11. Illustrates performance based on average time out of range for operational change periods. Higher scores indicate poorer performance. ............................................................ 18

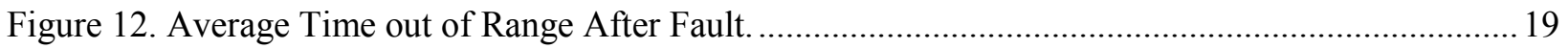

Figure 13. Average time out of range for each event type. Higher scores indicate poorer performance. Please note that the points represent the means of each of the values across all trials. The connecting lines represent change over time for the operational changes (the left three categories of time periods) but the faults did not always occur after the operational changes, therefore the line is not meant to represent a consecutive event.

\section{TABLES}

Table 1. Average time out of range in seconds for each automation type for Experiment Two 17

Table 2. Number of safety shutdowns in 508 trials. 18

Table 3. Average Time out of Range After Fault. 19

Table 4. Number of Safety Shutdowns. 20 


\section{ACRONYMS}

HAC Human Automation Collaboration

INL Idaho National Laboratory

LOA Level of Automation

NASA TLX National Aeronautics and Space Administration Task Load Index

SA Situation Awareness

SART Situation Awareness Rating Technique 


\section{Enabling Situation Awareness Under High Levels of Automation: Results From An Experimental Study \\ 1. INTRODUCTION}

The Department of Energy's Advanced Reactor Technologies Program sponsors research, development and deployment activities to promote safety, technical, economical, and environmental advancements of innovative Generation IV nuclear energy technologies. The Human Automation Collaboration (HAC) Research Project identifies developing advanced instrumentation and controls and human-machine interfaces as one of four key research areas.

New nuclear plants are expected to employ significantly more advanced technology than the systems in the current reactor fleet and utilize automation to a greater extent. However, advancing technology and increasing automation does not necessarily lead to improved efficiency and safety of the plant. A number of concerns about how these advanced technologies will affect human performance and the overall safety of the plant need to be addressed. More specifically, it is important to investigate how the operator and the automation work as a team to ensure effective and safe plant operation, also known as the human-automation collaboration.

The HAC Research Project at Idaho National Laboratory (INL) investigates collaboration methods between a system's automation and human operators and how that collaboration impacts the performance and reliability of overall system performance. Some well-documented human performance issues associated with typical HAC methods include reduced operator situation awareness (SA), complacency, and over reliance on the system automation (Endsley, 1995, 1996, 1997; Endsley and Kaber, 1999; Kaber and Endsley, 1997, 2004; Parasuraman et al., 2000; Sheridan, 2002; Wickens and Hollands, 2000; Wright and Kaber, 2005). The HAC Research Project addresses how to best design the collaboration between a system's automation and human operators in a manner that has the greatest positive impact on overall plant performance and reliability, hence addressing many of the documented issues. The ultimate goal of the HAC Research Project is to develop design guidance that supports optimal interaction between humans and automated systems.

In general, reliable automation improves system performance and supports safe and reliable production of electricity compared to manual performance. However, automation can have negative human performance consequences, such as complacency and human-out-of-the-loop problems. This tradeoff between automated system performance and human performance can be addressed in different ways. Many researchers advocate using intermediate levels of automation to handle these tradeoffs. However, this approach does not take full advantage of automation or human capabilities.

The researchers conducted an in-depth literature review to study current state-of-practice of HAC in the human factors and automation research fields, nuclear power industry, and similar process industries to identify knowledge gaps to address as a part of the HAC Research Project. The investigation of state-of-practice identified several important features of automation that influence the collaboration between automated systems and human operators, including; the level of automation (LOA), the cognitive function replaced by automation (e.g., detecting anomalies or taking control actions), adaptability, reliability, the process of automation, and automation modes. Oxstrand et al., 2013a, 2013b, and 2013c, provide more information related to the literature review and the development of the HAC model. 
The literature review found that many research studies demonstrated the effectiveness of adaptable automation in enhancing performance compared to manual designs. Some studies also demonstrated that automation fault recovery with adaptable automation is superior to fully automated systems. In addition, many studies have shown that SA is better with adaptable automation than with fully automated systems. Essentially, the promise of adaptable automation is a goldilocks balance that provides system performance similar to fully automated designs, but without the negative human performance consequences.

The HAC research team is investigating the use of adaptable automation as an alternative to the approach of using intermediate levels of automation. Adaptable automation is defined as a flexible or dynamic allocation of functions to human and automatic agents where the human is in control of the allocation. In contrast, adaptable automation is defined as flexible or dynamic allocation of function to human and automatic agents where the automated system is in control of the allocation. The adaptable approach may allow for higher levels of automation without sacrificing human performance (SA in particular), however previous research has been insufficient to confirm whether adaptable automation is more effective than intermediate levels of automation.

The experimental study focuses on the effect of LOA in SA and familiarization with the automated system. This report describes the relevant previous research activities and the current experiment in detail.

\subsection{Summary of Experiment One}

Although adaptable automation has been the focus of multiple research studies, there are many unanswered questions regarding the applicability and superiority of adaptable automation compared to intermediate automation. For example, few studies actually compare adaptable automation to intermediate automation levels. Instead, most studies focus on comparing the extremes of automation (manual and fully automatic). Thus, the question still remains; does adaptable automation manage human performance and system performance tradeoffs more effectively than static intermediate levels? INL researchers designed an experimental study to address this specific question.

In 2014, the research team conducted an experimental study comparing human performance and SA across four levels of automation using a simplified process control simulation. The study compared performance on a simplified process control simulation using an adaptable automation design (task delegation interface) to varying levels of automation under normal conditions and automation failure conditions.

\subsubsection{Process Control Simulation}

The majority of advanced reactors concepts are in the early design stage; therefore it is difficult to find a full-scale simulation model to use for testing human-automation interaction concepts. Furthermore, it is challenging to find trained operators to serve as participants in HAC research.

To facilitate research investigating general HAC principles in process control environments, the researchers developed a simulation mimicking the general structure of advanced reactors operations but with a more digestible surface structure such that non-operators could perform the task. The process control task was also designed to be difficult to ensure the measures used could detect differences in performance among participants and across levels of automation. The manual mode, for instance, required operators to take many actions per minute to keep all seven processes within prescribed parameters. 
In order to enable generalizability to an advanced reactor concept, the process was designed with proposed concepts of operations for small modular reactor (SMR) in mind. The process, for example, of managing two separate product streams (mixing tanks A and B) with different operational requirements is analogous to the proposed concept of using the SMR plant to supply both electricity generation and process heat. Also, the product streams have dynamic requirements, which is analogous to the operational concept of potential load following for SMR. Although the process control simulation incorporates these advanced reactor-like concepts of operation, mimicking actual nuclear processes was not the intent. Instead, the simulation was a mock chemical processing plant called SMR Chemical responsible for mixing and distributing Pantheradine.

The process control simulation enabled the researchers to investigate the interaction and collaboration in a controlled way that could also be generalized to the context of nuclear power plant operations. The process was designed to be simple enough for undergraduate psychology students to be able to operate with minimal training, but complex enough to allow for varying the LOA.

The four possible LOAs in the process simulation included:

1. Manual: The operator handles all tasks manually.

2. Intermediate: The automation monitors, generates responses, and presents possible actions to the operators, and the operator chooses the actions to be taken, the automation then carries out the chosen action.

3. Adaptable: The process was divided into four high level tasks. The operator can choose to delegate any (or all) of the four tasks to the automation or perform the tasks manually.

4. Fully Automatic: Automation handles all tasks, Operator task is to monitor and take manual control if necessary.

\subsubsection{Summary of Experiment One Methods and Results}

Experiment One participants 101 undergraduate psychology students at Idaho State University, out of which 35 were males and 66 were females. The average age was 23 years. The participants were given course credit for their participation.

The researchers hypothesized that the adaptable automation would yield the best performance and SA combination, but found there was no difference between intermediate and adaptable automation. Hence, the results from the experimental study do not confirm the common claim that adaptable automation is an optimal method to manage human performance and system performance tradeoffs associated with increasing automation. However, the results do not necessarily refute it either. Some methodological weaknesses in the study may have limited the applicability of the results to truly testing the effectiveness of adaptable automation for maintaining SA and enhancing system performance.

First, the study was conducted with university students who received minimal training. The fact that the participants were novices may have limited the degree to which they could achieve acceptable levels of SA. This may have contributed to the lack of differences in SA across the LOA conditions. Further, the task required continuous adjusting of the components, which may have overwhelmed participants and limited their ability to maintain awareness of the overall process.

Second, the process control simulation was not sufficiently complex to design a truly hierarchical abstraction scheme. The fact that the adaptable system was not sufficiently similar to other adaptable task delegation interfaces concepts may have limited the ability to detect differences between adaptable and intermediate LOAs. 
The inconsistencies between the results from the experimental study and existing literature indicates the need for additional research to investigate if adaptable automation in fact can enable higher LOAs without introducing human performance costs. It is yet too early to determine if adaptable automation is truly superior to intermediate LOA. To address the inconsistencies in the result and to address additional questions a second experimental study was conducted in 2015, as described in the next Section 3.

\section{EXPERIMENT TWO 2.1 Objective}

The purpose of Experiment Two was to further investigate the effects that LOAs have on system performance, human performance, SA, and workload by addressing some of the methodological challenges of Experiment One.

The main weakness of Experiment One was the fact that the participants were novices, which may have limited their SA and influenced their use of automation. Therefore, the main objective of Experiment Two was to ensure that all participants were proficient in managing the process before completing any experimental trials. This was accomplished in several ways. First the training materials (a self-paced Power Point Presentation) was improved and included more detailed information. Second, changes to the process control simulation enabled stabilization of the process, which reduced the number of actions the operator needed to take. Third, based on feedback from experiment and pilot participants, the user interface was redesigned to be more intuitive and usable (potentially reducing confusion associated with how to operate the equipment via the user interface). Fourth, the participants were trained to the criterion of being able to control the process without a safety shutdown for 10 consecutive minutes. Participants did not continue with experimental trials until they met this criterion. Finally, the experiment utilized a within-participants design (meaning that all participants were exposed to all LOA), which allowed the participants to gain considerable experience in controlling the process. Participants spent a total of 10 hours in Experiment Two (compared to just 1 hour in Experiment One).

Another issue with Experiment One was that the automation design was not an adequate analogy of the adaptable task delegation interface that was used by other authors investigating adaptable task delegation interfaces in previous studies (e.g., Miller and Parasuraman, 2007; Miller et al., 2005; Miller et al., 2003). The process control simulation was slightly modified to enable a more comparable adaptable automation condition.

\subsection{Method}

To investigate the affects automation has on overall human-system performance, researchers employed a simplified process control simulation using a nested experimental design to evaluate performance differences between four LOAs (manual, intermediate, adaptable, automatic) with varying faults and conditional changes occurring during each scenario. As mentioned earlier, some of the documented issues of automation include reduced SA, complacency, and over reliance on the system automation. Here, the effects of automation were measured by participant's ability to detect and respond to failing automation and conditional changes using a variety of metrics assessing SA, system performance, and workload. The intent of this study is to determine the goldilocks condition for HAC.

\subsubsection{Updates to Simulator}

An outcome of Experiment One was discovering the process simulator needed a more usable interface to make the process manageable and easier to understand. Experiment One also revealed the need to redesign the adaptable automation condition to more accurately match adaptable task delegation interfaces used in previous research.

One issue with Experiment One was the many participants that lost control of the process without ever regaining control for the duration of the scenario adding noise to the data. To address the issue 
Experiment Two added a function which reset the system if the participant lost control of the system. The system was considered 'out of control' if a process level, temperature, or concentration exceeded their high-high or low-low thresholds. The system reset would notify the participant a reset had just occurred while returning the system to a neutral status and allowing the participant to continue. It is also possible that participants in Experiment One did not receive adequate training on system recovery thus their consistent inability to do so. More in depth training was added to Experiment Two as a precaution. The reset is recorded in the exported data to enable tracking of how many times the system reset during a scenario.

The addition of the system reset in the second experiment served a dual purpose. First it allowed participants to continue the process control simulation in a controlled manner providing more performance data. Second, logging the number of system shutdowns provided a metric for determining participant's ability to handle the system in general.

The process simulator was further revised by removing the control buttons for the pumps and valves. Instead of buttons the participant operates the valves and pumps (as seen in Figure 1) by clicking on the control to toggle it on or off (See Figure 2). The capital letter 'A' would appear on pumps and valves set to automatic for distinguishability. Removing the buttons freed space allowing for a cleaner user interface. Experiment Two provided participants the capability to adjust all pump speeds allowing finer control of the concentration in the mixing tanks. Lastly, accumulator B was removed after being determined unnecessary. Again, removing it simplified the interface by removing clutter.

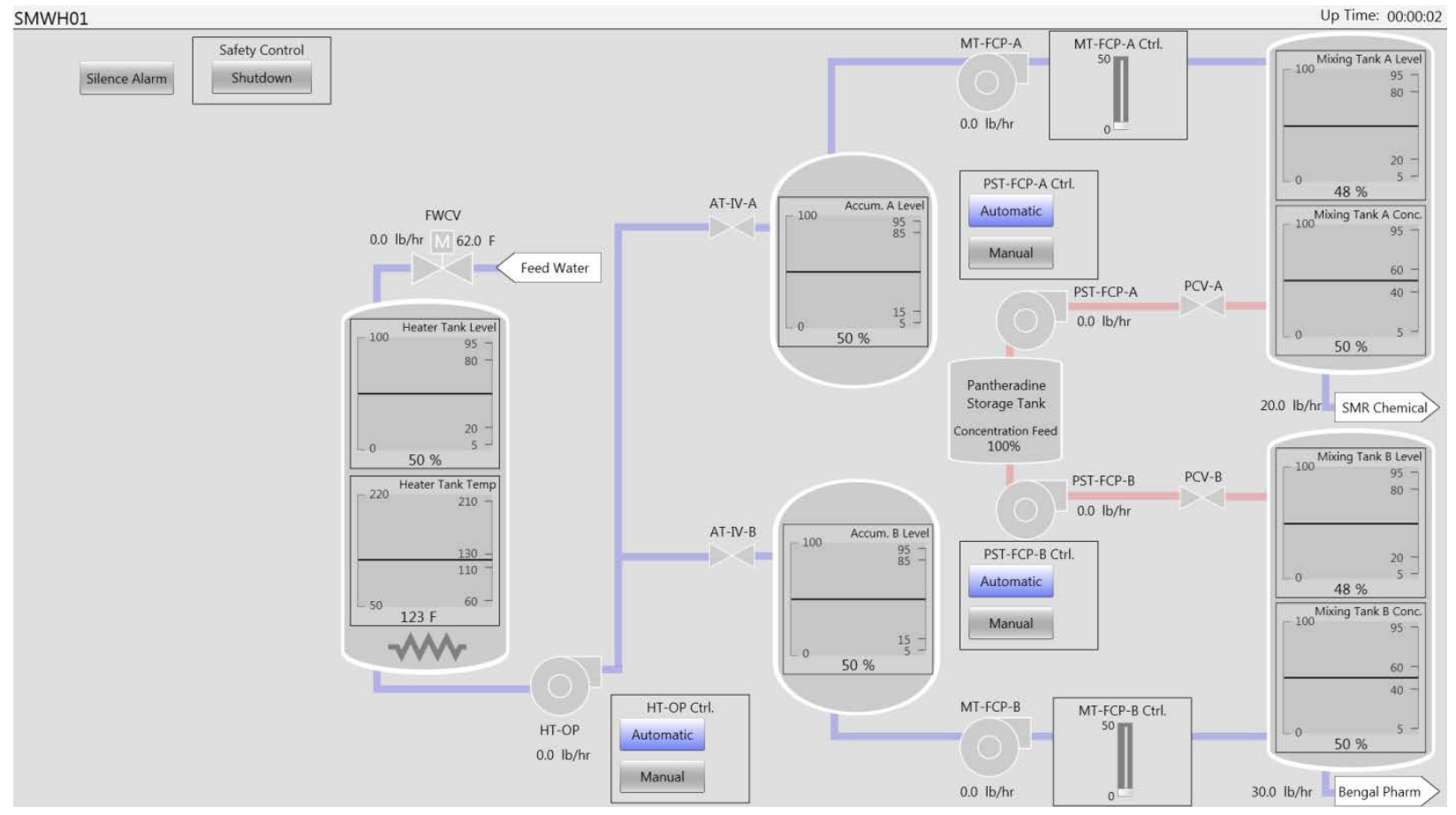

Figure 1. Interface used in Experiment One. 


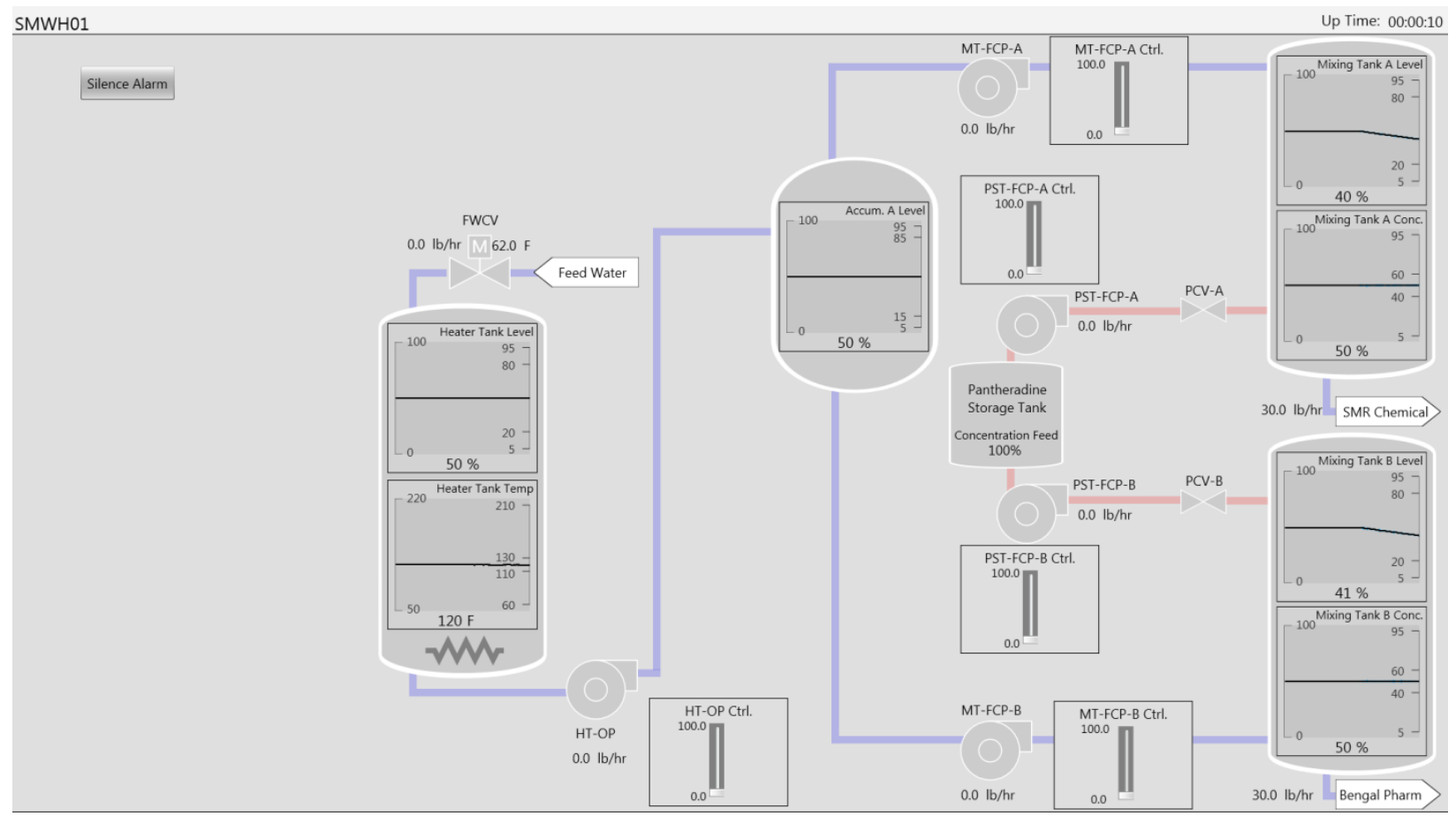

Figure 2. Interface used in Experiment Two.

\subsubsection{Task Description}

Participants were asked to assume a "process operator" role for the pseudo company called SMR Chemical. SMR Chemical is responsible for distributing a pseudo chemical known as "Pantheradine" at prescribed concentration levels and production rates (pounds per hour). Customers provide the desired Pantheradine concentration and the rate in pounds per hour that they wish to receive the chemical mixture. The participant's role as process operator was to meet the requirements of the customer by monitoring and maintaining a sufficient supply of properly concentrated Pantheradine to two separate mixing tanks (one per customer). Figure 3 displays the control and tank configuration the participant used to achieve their goal as process operator. 


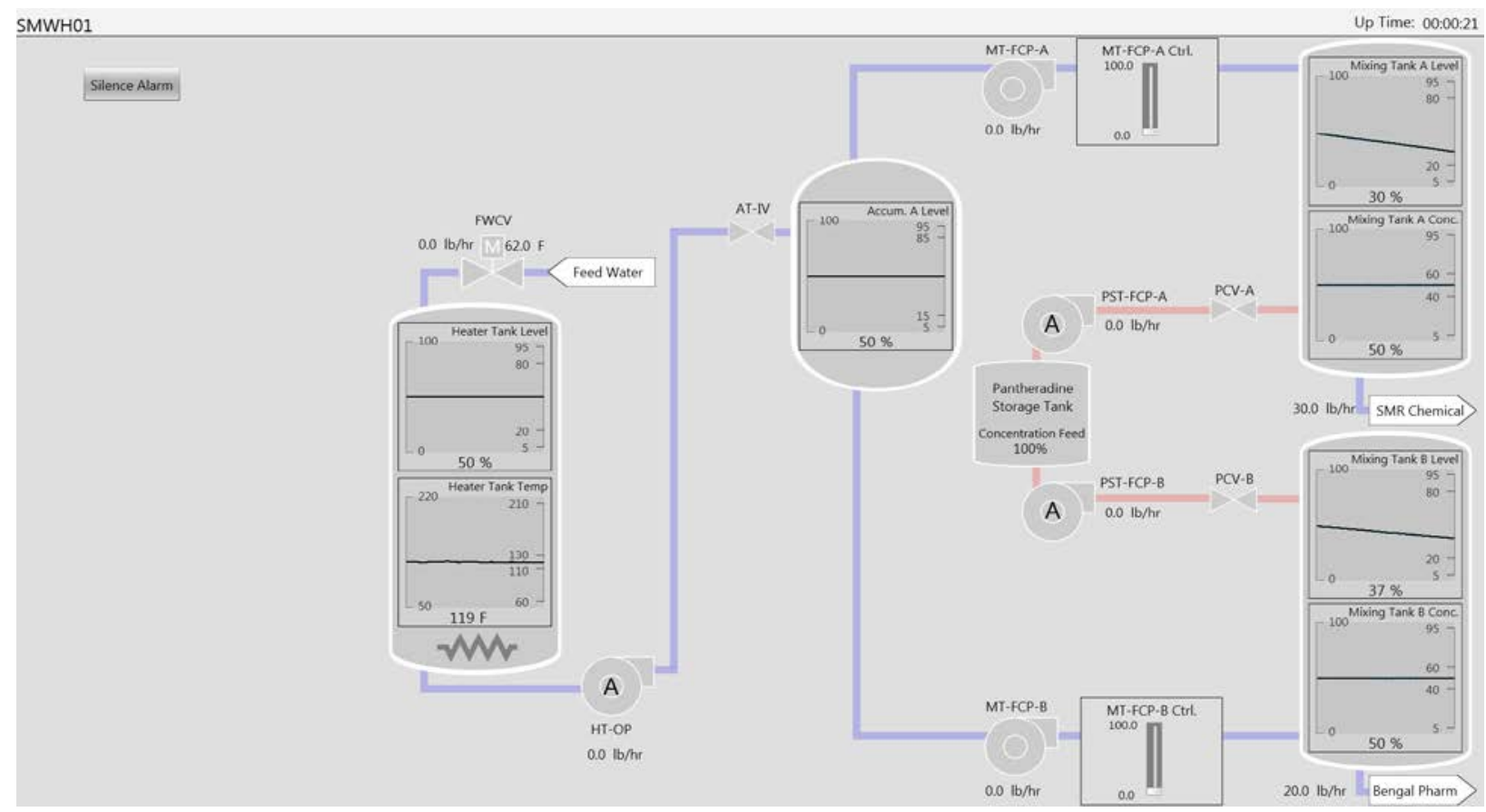

Figure 3. The control tank configuration of SMR Chemical.

The process operator must monitor and maintain a total of seven processes that include:

1. Heater tank fill level

2. Heater tank water temperature

3. Accumulation tank fill level

4. Mixing tank A fill level

5. Mixing tank A concentration level

6. Mixing tank B fill level

7. Mix tank B concentration level

The mixing process depicted in Figure 3 moves left to right beginning with the heater tank. As the name suggests, the heater tank's purpose is to raise the feed water temperature to between 110 and 130 degrees Fahrenheit. The process operator must keep the tank supplied with feed water while maintaining the correct water temperature. Opening the feed water control valve will affect change to both heater tank parameters: adding water will increase the tank fill level (rate of fill dependent on out flow) and lower the temperature of the existing tank water. The operator can counteract the cooling effect of new water by activating the heating coil at the bottom of the heater tank display. The process operator must successfully maintain the heater tank level and water temperature by using the feed water control valve to add feed water and the heater coil to heat new water.

The heated water then flows to an accumulation tank. The accumulation tank is an intermediary storage tank that supplies both mixing tanks with hot water. The process operator must maintain the fill level of the tank within provided parameters by regulating supply from the hot water tank with a valve and pump. The accumulation tank inlet valve operates as a supply on/off switch while the heat tank outlet pump offers variable control over flow rate from heater tank to accumulation tank.

The final step of the mixing process is managing the fill level and concentration percentage of mixing tank A and B. The fill level of each mixing tank is replenished by the aggregate input from the 
accumulation tank (hot water) and Pantheradine tank (chemical). Each tank is depleted at a rate set by each customer ( 1 customer per tank). The concentration percentage of each mixing tank is dependent on the ratio of hot water and Pantheradine input. Two pumps supply each mixing tank; one for hot water from the accumulation tank, one for the Pantheradine. Using these pumps the process operator can monitor and manage the aggregate fill rate and proper supply ratio to ensure customer demands are being met.

To monitor each process a display with tick marks , numerical tank status indicators, and trend displays are associated with each process and located on each tank using the principle of proximity in design for easy association and detection process statuses.

Referring to Figure 4, the inner tick mark values on each tank indicate the range the process operator is to keep the tank within at all times and may refer to heat, fill, or concentration level. The current tank value is redundantly displayed as a continuous trend line on the tank and a numerical display. Additionally, a visual alarm highlights any parameter display that has been violated. Despite this assistance, managing all seven processes individually was considered a manual task. However, the process operators were not always required to complete their tasks using a manual interface.

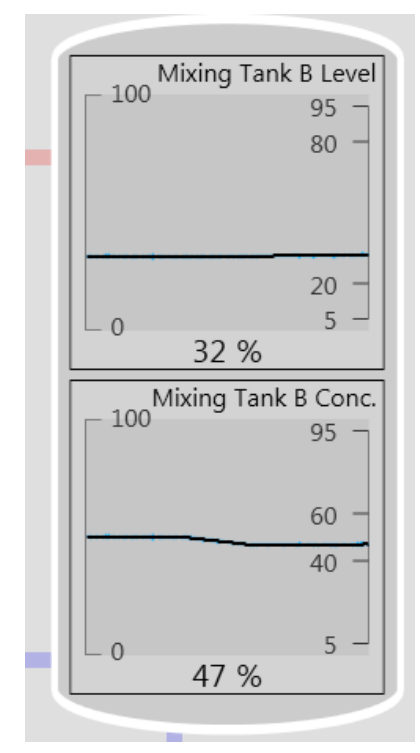

Figure 4. Numerical tank status indicators.

\subsubsection{Automation Descriptions}

Four LOAs were tested: Manual, Intermediate, Adaptable, and Automatic. Each LOA required different amounts of participation from the process operator. These four levels of automation are described below.

2.2.3.1 Manual. The manual condition requires the process operator to manage all seven processes individually. The operator must turn off and on valves, set pump flow rates, and heat the water to maintain the designated system parameters. No assistance outside the visual alarms and displayed trend lines and tank values was provided. See Figure 5 for an example of how the interface is presented. 


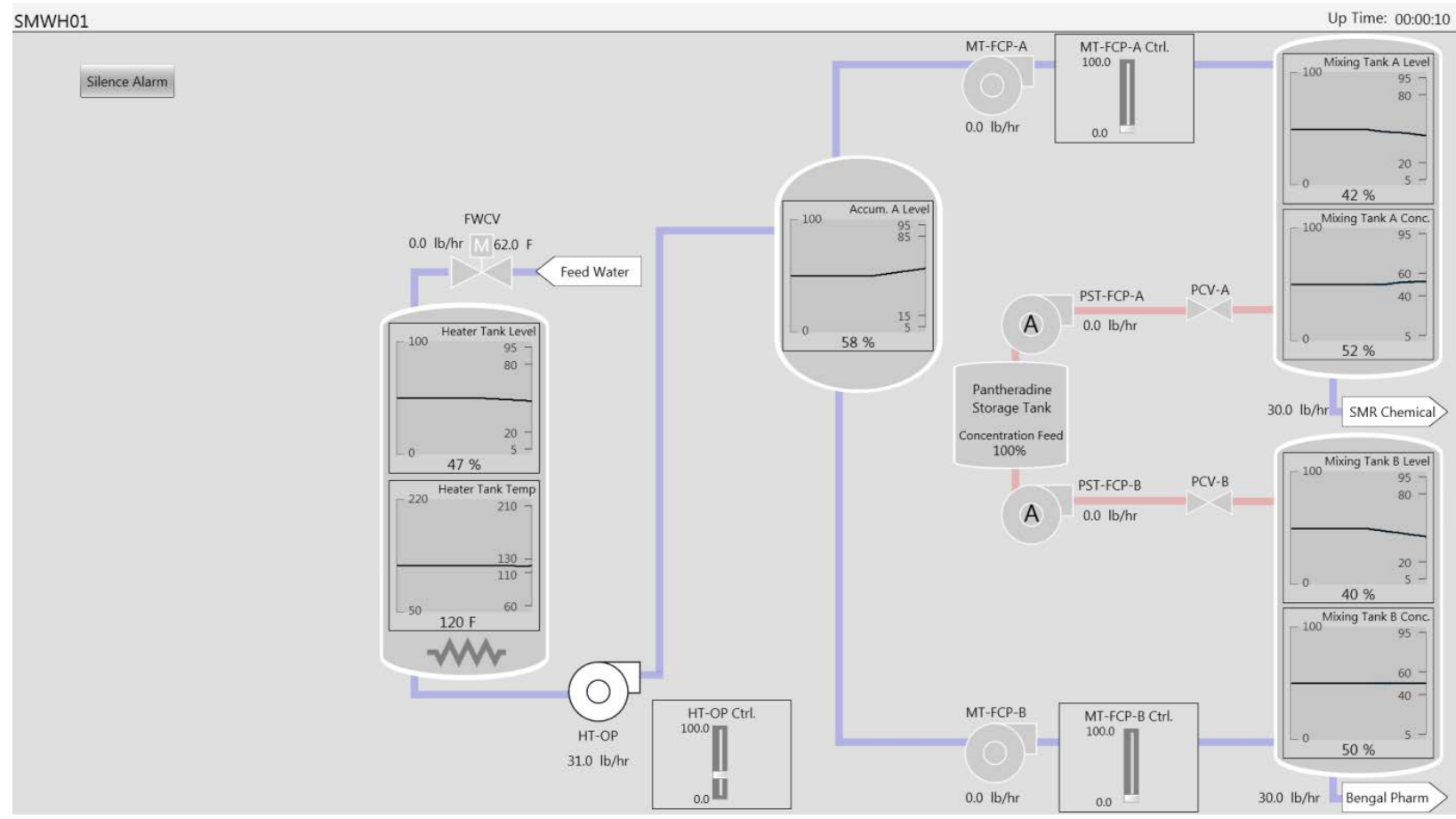

Figure 5. Manual Level of Automation.

2.2.3.2 Intermediate. The intermediate is modeled after Endsley and Kaber's (1999) fifth LOA in their "Level of Automation Taxonomy," which is described as:

"The computer generates a list of options that the human can select from; the operator may still generate his or her own options. The computer is responsible for implementing the chosen action. This LOA is common in many expert systems or decision support systems in which the operator may use or ignore the option guidance provided by the system."

The intermediate LOA in the context of nuclear power falls under O'Hara et al.'s (2010) level three "operation by consent" of the "Preliminary Levels of Automation for Nuclear Power Plant Applications". The automation and the process operator both monitor the system status when the intermediate LOA is engaged. The operator can continue to make manual adjustments as necessary. However, if a process begins to move outside the designated parameter an alert is activated. The alert is displayed on the left side of the screen with three components: 1) the solution, 2) an estimated time-to-event, and 3) a confirm or disregard option as displayed in Figure 6. Selecting 'confirm' signals the automation to carry out the prescribed solution. Selecting disregard hides the prompt. The operator could fully rely on the intermediate LOA without ever making a manual correction, or fully disregard the INTERMEDIATE and control the system as in the manual condition or any ratio in between. 


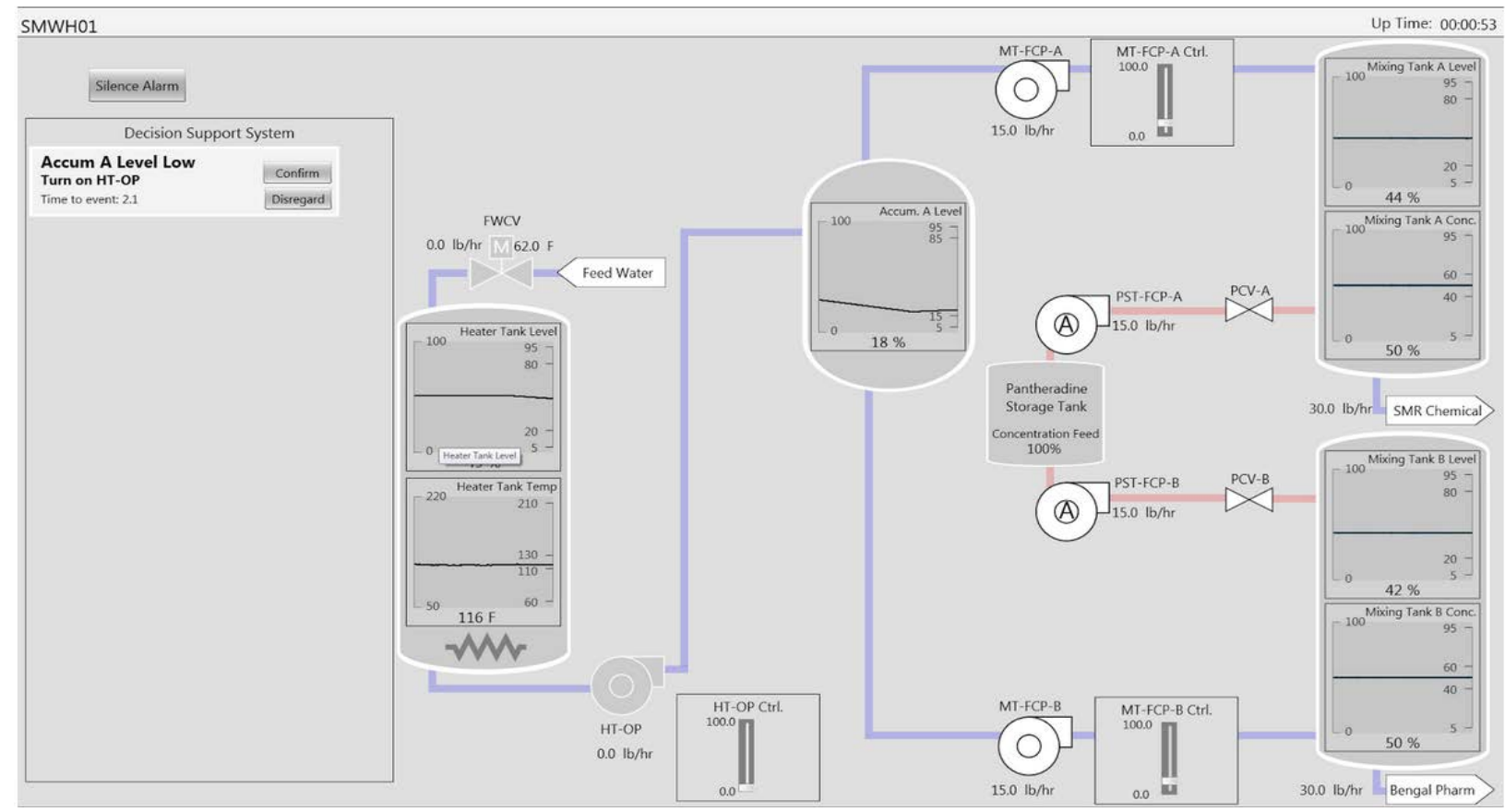

Figure 6. Intermediate Level of Automation.

2.2.3.3 Adaptable Automation. The adaptable LOA batched processes into four sections and provided a task delegation interface allowing the process operator to select one or more of the following batches to automate:

1. Heater Tank Management (2 processes)

2. Accumulator Tank Management (1 process)

3. Mixing Tank A Management (2 processes)

4. Mixing Tank B Management (2 processes)

However, the delegation interface was organized in a sequential hierarchy restricting certain combinations from use. The operator could delegate any process to automation as long as the preceding process was also automated, for example; if the accumulator tank was delegated to automation, the Heater Tank must also be automated. Processes occurring in parallel, such as the mixing tanks, could be automated separately or together.

The task delegation interface was presented on the left side of screen, the same place the prompts for the intermediate LOA are located. Two buttons for each batched section were labeled ' $\mathrm{M}$ ' for manual or 'A' for automatic with the active button highlighted in blue. If the automation was activated a gray box would appear outlining the activated section. Otherwise the interface is identical to the manual condition. The process operator using this LOA has the choice to control all systems manually, or automate the entire process. See Figure 7 for an example of how the interface was presented. 


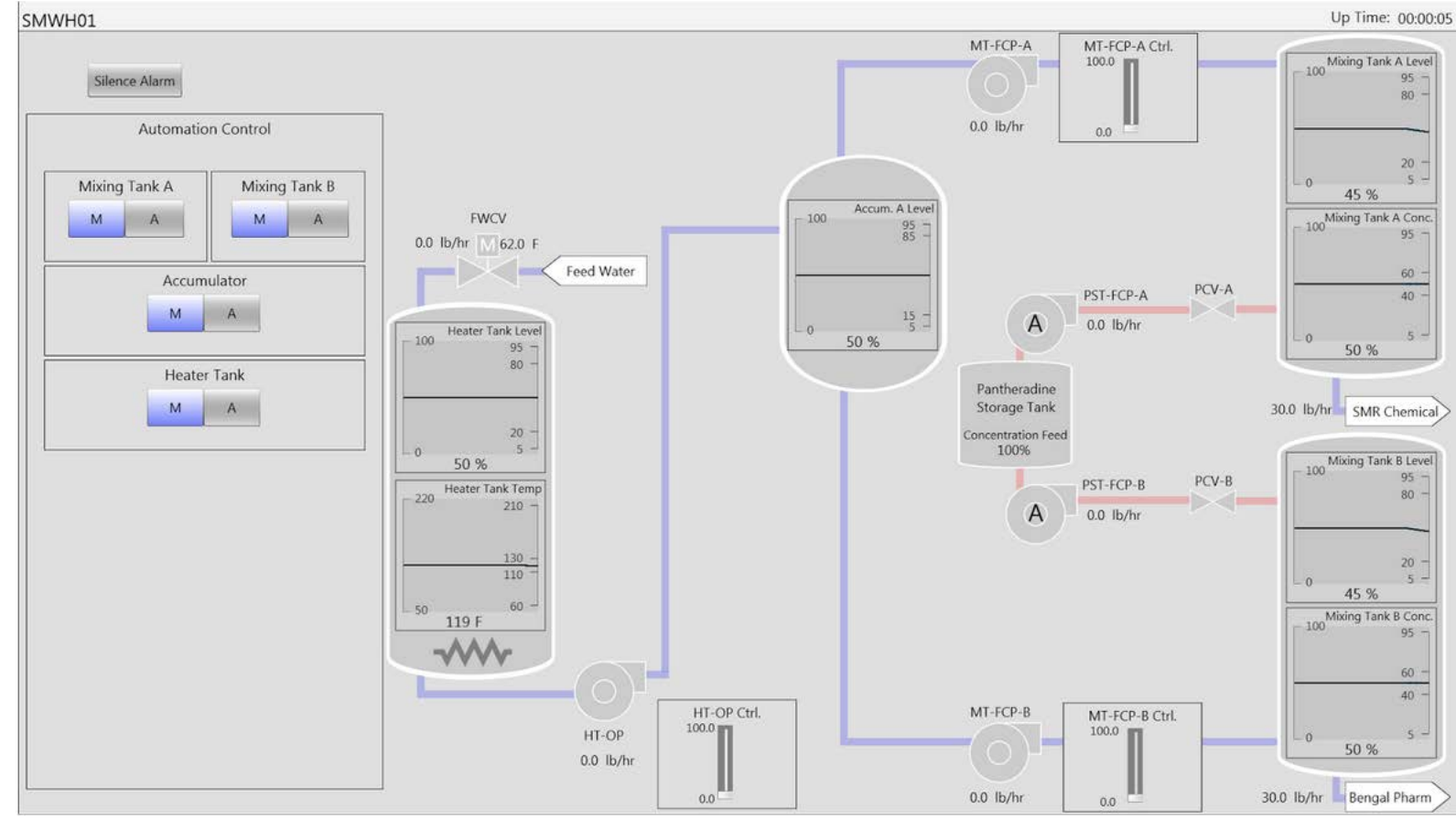

Figure 7. Adaptable Level of Automation.

2.2.3.4 Automatic. The Automatic LOA, as the name implies, automates the entire process requiring the operator to monitor the system taking action only when the automation fails. However, the operator has the option to switch any valve or pump from automated to manual by right clicking the equipment of choice and selecting 'manual'. This switch is easily reversible by carrying out the same action and selecting 'automatic' instead of 'manual'. See Figure 8 for an example of how the interface was presented.

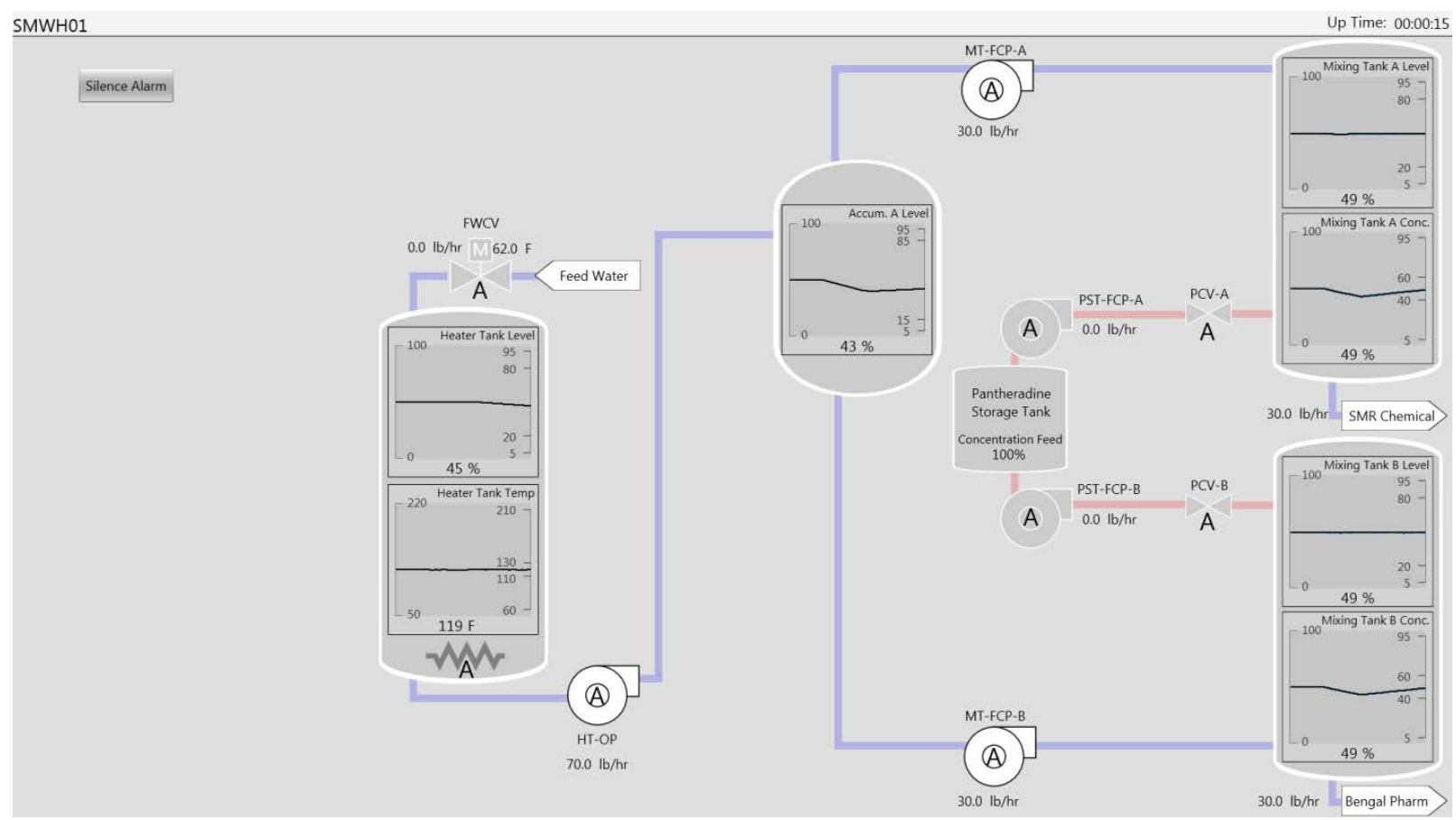

Figure 8. Automatic Level of Automation. 


\subsubsection{Participants}

Experiment Two participants included 16 INL employees recruited through an advertisement on a laboratory-wide daily distribution of iNotes (daily laboratory e-mail containing news, updates, and notices). Compensation was provided by paying the employees their hourly rate for the time spent participating in the experiment. There were 5 females and 11 males. Ages ranged from 19-63 years. The average age was 38 years. 4 participants reported previous process control operator experience, 12 did not.

\subsection{Experimental Design}

This experiment was designed to observe the effects of one particular independent variable, the LOA. Participants experienced each of the four LOAs once per experimental session. The order of presentation was derived for each participant using a pseudo random process to mitigate any possible order effects.

The second and only other independent variable was scenario construction. However, while this variable was manipulated within each participant, it was designed such that each of the four constructions was comparable to the next. The intent behind varying scenario construction was to avoid predictable fault occurrences and product demands from artificially inflating participant performance. Having four comparable scenarios helped create a realistic environment in which arising issues within the system came as a surprise rather than an expectation forcing participants to respond retroactively rather than proactively. A pseudo random pairing of scenario construction and LOA also mitigated any order effects or predictable patterns across the four experimental sessions within a participant.

\subsubsection{Independent Variables}

The four LOAs constituted the first independent variable: manual, intermediate, adaptable, and automatic. All four conditions were administered within participants. A summary of the automation conditions is as follows:

1. Manual: Participant monitors and manages all seven processes.

2. Intermediate: Participant monitors and manages all seven processes while automation provides support with prescribed solutions and the option to delegate the solution action to automation.

3. Adaptable: The seven processes are batched into four groups. Participant monitors all seven processes but can delegate automation to manage either all or none of the batched processes.

4. Full Automation: Automation manages all the processes while the participant monitors the processes only intervening when the automation fails.

Each scenario contained two event categories: an operational change and a fault event. An event from both categories occurred twice per scenario for a total of four events. Each 20-minute scenario was broken down into 5 minute blocks during which one of the following events would occur:

- Operational Change in mixing tank A or B

- Fault on concentration pump A or B

An operational change is intended to simulate normal operating conditions, and reflect a change in demand from one of SMR Chemical's customers requiring the process operator to make proportional adjustments to the mixture concentration and flow rate. The demand may change in one of two ways:

1. $10 \%$ demand increase or decrease to the current concentration level of a mixing tank

2. $10 \mathrm{lb} / \mathrm{hr}$ demand increase or decrease to the current output flow rate.

A fault is intended to simulate an equipment malfunction requiring the process operator to override automated control, if it is not already in manual, and readjust the value setting to accommodate the fault 
until notification of a fix is given. However, the system must currently be within all parameters for a fault to initiate to avoid overwhelming an already failing system. Faults can occur as two possible events:

1. Automation failure requiring manual override

2. Pump begins decreasing flow rate without explicit indication.

- If the pump is in manual mode the flow rate must be adjusted

- If the pump is in automatic mode, the pump must be manually overridden and the flow rate adjusted.

The faults were designed such that no matter what the conditions before the fault, the fault would affect the process and require operator intervention to maintain the process requirements.

Since evaluating how scenarios affected system performance was not of interest, each scenario was designed to be comparable to the next. Hence, each scenario was broken down into 5-minute trial blocks during which one of the above events would occur. Furthermore, one constraint was placed on the pseudo random pairing of scenarios to the LOA that ensured that equal numbers of each event occurred during a single LOA across all four experimental sessions, that is, 8 blocks contained faults and 8 blocks contained operational changes during the 80 minutes a participant spent in a single LOA overall.

Overall, each participant was exposed to 16 fault events and 16 operational change events per automation condition across four sessions.

\subsubsection{Dependent Variables}

A battery of measurements was collected to capture operator and automation performance. Objective data were collected by sampling the current status of the process control task approximately once every 5 seconds $(0.2 \mathrm{~Hz})$. Logged with each sample were the state of all seven parameters, operational requirements, faults present, whether a safety shutdown occurred, time a parameter has remained out of range, and current setting of all pumps and valves. Subjective assessment of workload, SA and trust were collected via the National Aeronautics and Space Administration Task Load Index (NASA TLX) freeze-probe questionnaire, the Situation Awareness Rating Technique (SART) and a post hoc questionnaire.

\subsubsection{System Performance}

System performance comprises the human and automated system performance in accomplishing the overall process goals. In this case, system performance represents the ability of the human-system to provide the correct concentration of Pantheradine to both processes while maintaining a safe system state. System performance was assessed by measuring the time each of the process parameters was out of range. A secondary measure of system performance was the number of safety shutdowns that occurred. The scenarios were broken down into trial periods in which either an operational change had occurred or the time period immediately after a fault was injected.

Performance measures for operational change periods included the:

- Number of safety shutdowns

- Time any parameters was out of range

Performance measures for fault response periods included the:

- Number of safety shutdowns

- Time out of range during fault response period 


\subsubsection{Human Performance}

Researchers assessed human performance by measuring SA and workload. SA was assessed using a short freeze-probe questionnaire. Three times during each 20-minute scenario, the screen simulation would freeze and present an SA questionnaire to the participant. The freeze-probe questionnaire assessed current state awareness by asking which (if any) of the parameters were currently out of range, and assessed past safety awareness by asking if any events had occurred in the past 3 minutes (an equipment malfunction, a safety shutdown, or an operational change). See Figure 9 for an example of how the SA questionnaire was presented to the participants. Following each scenario, the participant was asked to respond to two questionnaires. The SART questionnaire was a secondary subjective measure of SA and the NASA TLX assessed workload.

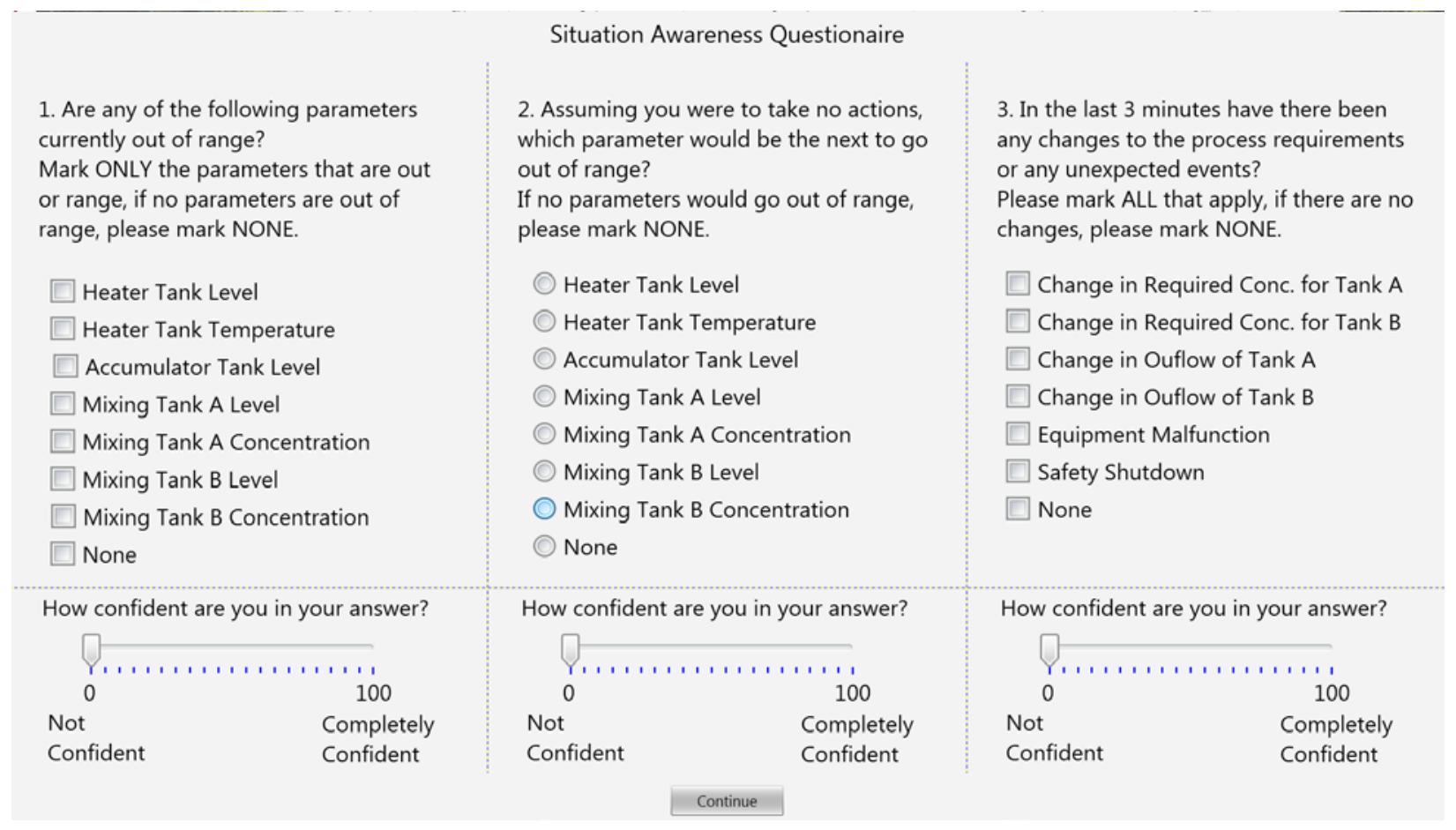

Figure 9. Freeze-probe Questionnaire.

\subsubsection{Experimental Protocol}

Participants were first given a consent form to read and sign and encouraged to ask any questions on items that may be unclear on the form. After a signature was provided, the participants were given a brief description of how the next 5 sessions were to be structured and what to expect. A demographic questionnaire was then completed and the study began. Refer to Figure 10 for a graphical breakdown of the 5 sessions and what each included. 


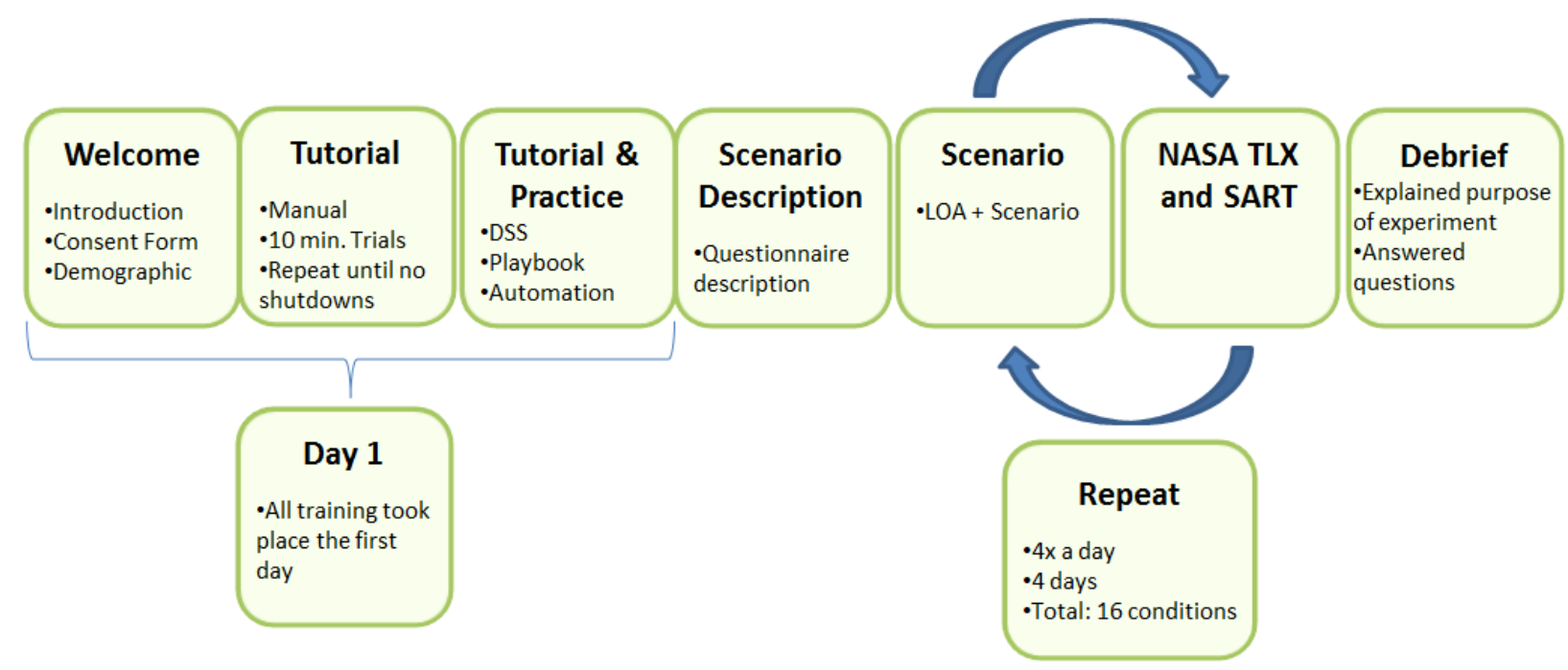

Figure 10. Experimental Protocol.

The first day following the demographic questionnaire was dedicated to training. The participant received a Microsoft Power Point tutorial to learn their role as process operator. Participants were encouraged to ask questions if anything was unclear. The training described the system goal and how to identify and use all the equipment which included:

- How to control manual/automatic pumps

- How to switch valves on/off

- How to read the tank displays

- Purpose of each tank, pump, and valve.

The training also covered basic operations best practices such as:

- Meaning of alerts and warnings

- Safety shutdown procedure

- How to stabilize the mixing process.

Following the tutorial was a 10 minute manual LOA condition session that served the dual purpose of practice and litmus test. The session allowed participants to gain familiarity with the system and ask any further questions. However, participants were required to complete a full 10-minute session without a shutdown before continuing. No faults or operational changes were introduced at this point. This requirement ensured participants were competent with the system before beginning the experimental sessions.

Once an entire trial period was completed without a system shutdown the participant could continue to train on the other three LOAs. A 10-minute practice session followed the tutorial on each LOA to gain proficiency with LOA interface. The first day was concluded once all tutorials and LOA practice sessions had been completed. 
Upon arrival to each of the following experiment sessions, participants was given the opportunity to ask any questions or run a 5 minute refresher trial as practice before beginning the experiment. If they declined or once the practice was completed they received a brief description of what was expected during that day and the session commenced. During each session the participant received one 20 minute trial of each LOA totaling approximately 80 minutes of simulation run time. During each trial a freeze-probe questionnaire would pause the simulation only resuming once the participant answered all three questions. The NASA TLX, SART and Trust questionnaire were administered after each 20 minute trial.

\subsubsection{Hypotheses}

The literature on human automation interaction predicts that intermediate automation will lead to an acceptable combination of human and system performance. That is, intermediate automation will lead to adequate system performance under anticipated conditions (but not as good as fully automatic performance), and adequate performance under unanticipated conditions such as automation failures (due to the fact that SA is not as degraded as it would be under fully automatic conditions). However, other research suggests that optimal performance may be achieved by flexibly allocating automation using an adaptable task delegation interface. Experiment One revealed that there might not be a difference between performance using a typical intermediate LOA and an adaptable LOA. Further, the authors of Experiment One concluded that because participants delegating a similar number of total actions to the automation under conditions of intermediate and adaptable LOAs, the two LOAs were too similar to be characterized as distinctly different LOAs. Methodological challenges present in the experiment limited the degree to which generalizable conclusions could be drawn from the results of Experiment One. Experiment Two was designed to address the methodological challenges and assess whether there is in fact a benefit of adaptable automation compared to an intermediate level.

System performance during normal operational changes (i.e., concentration and outflow changes in the mixing tanks) as assessed by time out of range and number of safety shutdown will show the following pattern:

- Automatic $>$ Adaptable $>$ Intermediate $>$ Manual

System performance during fault periods as assessed by time out of range and number of safety shutdown will show the following pattern:

- Manual $>$ Adaptable $>$ Intermediate $>$ Automatic

SA as assessed by the freeze-probe questionnaires will show the following pattern:

- Manual $>$ Adaptable $>$ Intermediate $>$ Automatic

To summarize, during normal operations (such as during an operational change, or during steady state operation) system performance will be best under fully automatic conditions, and worst under manual. If adaptable automation is superior to intermediate LOAs then system performance will be better with the adaptable automation than the intermediate level. For fault periods and SA, the relationship is expected to invert for manual and fully automatic levels of automation, because human monitoring and ability to regain manual control of the process is typically best under more manual conditions. Similarly, if adaptable automation is better for managing human performance tradeoffs of automation, then fault performance and SA should be better for adaptable than for intermediate LOAs.

\subsection{Results and Discussion}

The system performance scores were evaluated separately for operational change periods and fault periods. 


\subsubsection{Operational Change Performance}

A typical participant experienced $3210 \%$ changes in desired concentration and flow volume (one in each of two mixing tanks per 20-minute scenario) over the course of the study. One full minute was allowed to implement each concentration change: participants received a popup message informing them that the old production would stop in 10 seconds and a new production would begin in 1 minute. That is, for 50 seconds, no outflow occurred from the mixing tank, during which time the participant could do whatever was necessary to achieve the new concentration target.

2.4.1.1 Time out of Range. Performance was evaluated during three 1-minute periods: the minute prior to the notification of an impending change; the minute between notification and restarting production, and the first minute after production resumed. The minute before establishes a participant's baseline performance level; the middle transitional minute measures how efficiently the participant adapted to the new demands; and the following minute, whether the participant succeeded in regaining steady control of the process after responding to the changes. Perfect performance during the transitional period was not expected; rather, "success" might be defined as having all parameters in range at the time production resumes, and by that measure, participants successfully handled $91 \%$ of the concentration-change challenges.

Participants generally had the process under control prior to the concentration change,; experienced a mild degradation in performance during the transitional period,; and performed slightly worse after the transition period than they did before it. Averaged across all participants and trials, the process was out of bounds range an average of 2.2 seconds ( 0.2 seconds for the best participant, 10.1 seconds for the worst) in the minute preceding a concentration change,; 7.4 seconds ( 2.0 to 18.4 seconds) during the transition period,; and 4.5 seconds ( 0.1 to 14.0 seconds) in the following minute.

For normal operational changes, the automated system had the ability to help the operator adapt to a change in desired concentration. Consistent with that fact, participants using the automation had better success at maintaining control of the process. Table 1 shows the average time out of range for each automation type.

Table 1. Average time out of range in seconds for each automation type for Experiment Two.

\begin{tabular}{|l|c|c|c|}
\hline \multicolumn{1}{|c|}{ Automation type } & $\begin{array}{c}\text { Before } \\
\text { (seconds) }\end{array}$ & $\begin{array}{c}\text { During } \\
\text { (seconds) }\end{array}$ & $\begin{array}{c}\text { After } \\
\text { (seconds) }\end{array}$ \\
\hline Manual & 5.9 & 10.6 & 8.0 \\
\hline Intermediate & 1.8 & 8.1 & 6.7 \\
\hline Adaptable & 0.8 & 6.4 & 2.2 \\
\hline Automatic & 0.2 & 4.3 & 1.0 \\
\hline
\end{tabular}

Figure 11. Illustrates performance based on average time out of range for operational change periods. Higher scores indicate poorer performance. It is clear that automation enhances performance during all three periods of the operational changes. 


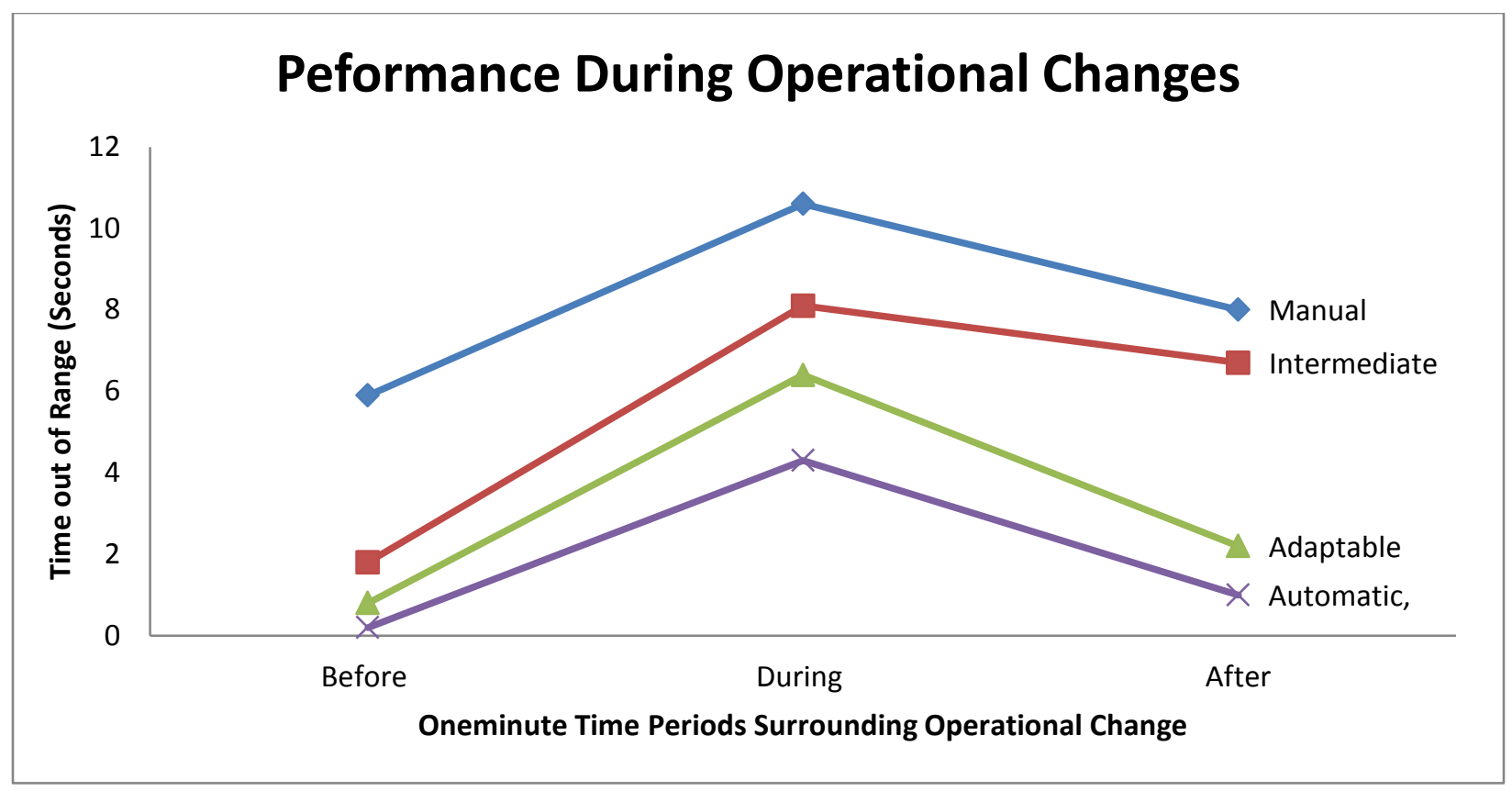

Figure 11. Illustrates performance based on average time out of range for operational change periods. Higher scores indicate poorer performance.

2.4.1.2 Learning Effects. Performance improved slightly from day to day (6.1, 5.8, 3.6, and 2.4 seconds out of range in the minute after production resumed, on the first through forth day, respectively.)

2.4.1.3 Safety Shutdowns. Safety shutdowns were rare for all participants (five, five, and seven, before during and after the transition period, in 508 trials, with no one participant having more than two shutdowns). Table 2 summarizes the number of safety shutdowns for the operational change periods.

Table 2. Number of safety shutdowns in 508 trials.

\begin{tabular}{|l|c|c|c|}
\hline \multicolumn{1}{|c|}{ Auto Type } & Before (seconds) & During (seconds) & After (seconds) \\
\hline Manual & 2 & 2 & 5 \\
\hline Intermediate & 3 & 0 & 0 \\
\hline Adaptable & 0 & 2 & 2 \\
\hline Automatic & 0 & 1 & 0 \\
\hline
\end{tabular}

\subsubsection{Fault Response Performance}

A typical participant experienced 32 pump faults (two per 20-minute scenario) over the course of the study. Performance was evaluated by observing how many safety shutdowns occurred in the minute prior and the minute following fault injection, and observing how much time at least one parameter was allowed to go out of range. 
2.4.2.1 Time Out of Range. There were strong differences among participants, but all participants performed generally well prior to injection of a pump fault, and all suffered a detectable degradation in performance following the fault.

The best participant averaged less than 0.1 seconds out of range in the minute prior to a pump fault, and 2 seconds in the minute after; the worst participant, 3 seconds prior and 35 seconds after. The average across all participants was 1.1 seconds in the minute prior and 10.8 seconds in the minute after. Only one participant had a safety shutdown in the minute prior to a pump fault. Performance after a pump fault also varied strongly with automation type, with participants already involved in manual control of the process better able to cope with a malfunction. Table 3 summarizes the average performance after the fault was injected.

Table 3. Average Time out of Range After Fault.

\begin{tabular}{|l|c|}
\hline \multicolumn{1}{|c|}{ Automation type } & Average time of out range (seconds) \\
\hline Manual & 5.8 \\
\hline Intermediate & 7.0 \\
\hline Adaptable & 11.9 \\
\hline Auto & 16.7 \\
\hline
\end{tabular}

Figure 12 Illustrates the average time out of range after a fault. During this period, it appears that automation degrades performance compared to manual control.

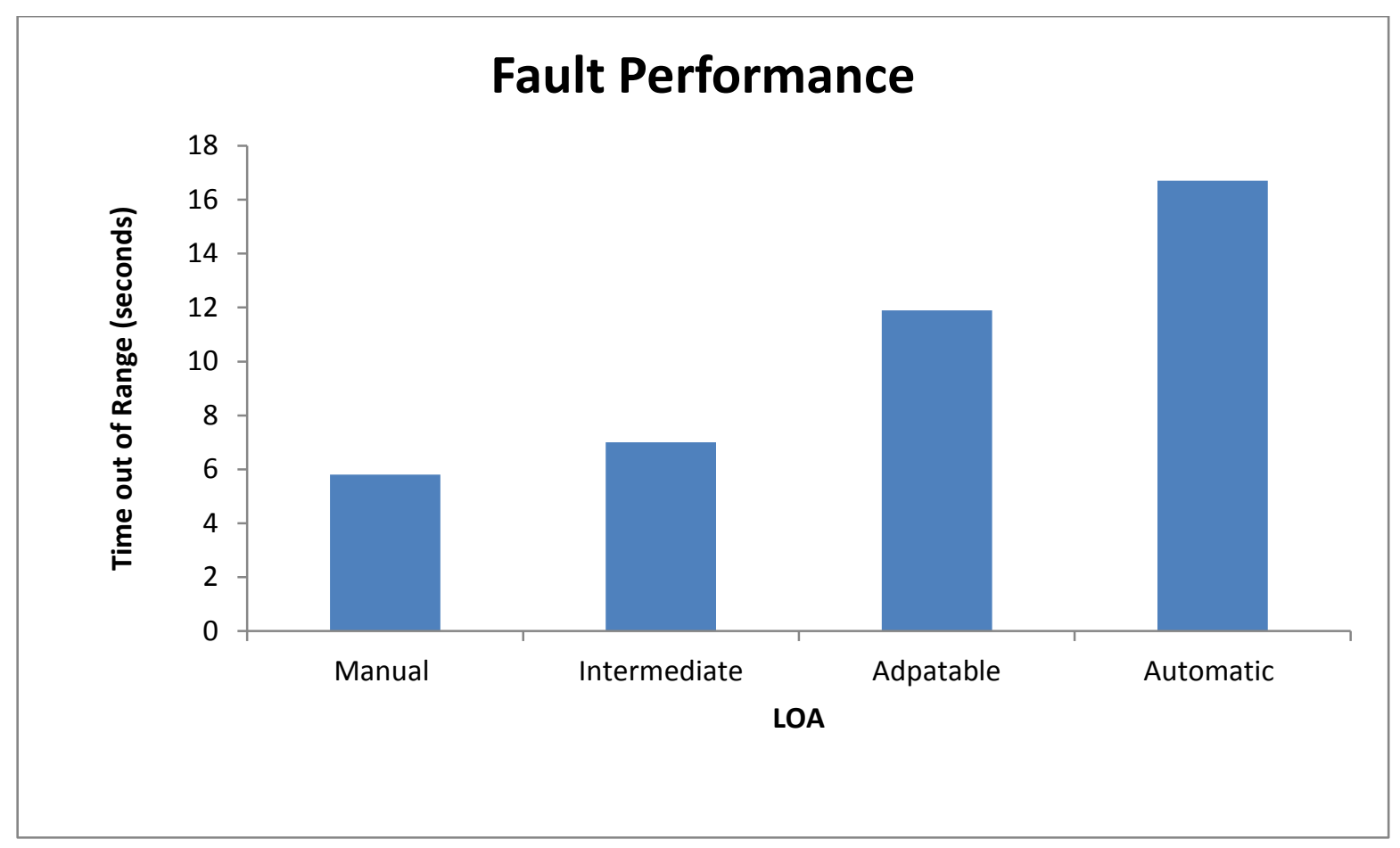

Figure 12. Average Time out of Range After Fault. 
2.4.2.2 Safety Shutdowns. After a fault, the overall rate of safety shutdowns was 0.1 per minute. Only three participants avoided ever having a safety shutdown, and five only had one safety shutdown. The worst participant had 23 safety shutdowns in 29 trials ( 0.79 per minute). Table 4 summarizes the safety shutdowns based on LOA.

Table 4. Number of Safety Shutdowns.

\begin{tabular}{|l|l|}
\hline Automation type & Average number of safety shutdowns \\
\hline Manual & 0.06 \\
\hline Intermediate & 0.06 \\
\hline Adaptable & 0.11 \\
\hline Automatic & 0.13 \\
\hline
\end{tabular}

2.4.2.3 Learning Effects. Participants continued to get better at responding to faults over the course of the study, despite having been "trained to competence" for a day prior to beginning scored trials. Average time out of range after a pump fault was 15.1, 9.8, 9.0, and 8.1 seconds, on first through fourth day operating the process. Within a single day, an interesting combination of learning and fatigue effects was visible: participants tended to perform worst on their first trial of the day and best on their third. sufficient data were not collected to formally investigate the interaction between the day, scenario number on a given day, and timing early or late in a single scenario.

\subsubsection{Situation awareness}

Situation Awareness was assessed objectively for the current and past states of the process by analyzing the performance on the freeze-probe questionnaire. Subjective SA was assessed by analyzing the responses to the SART questionnaire.

2.4.3.1 Freeze-Probe Questionnaires. Question 1 on the freeze-probe SA questionnaire asked participants which of the seven process parameters (four tank levels, one tank temperature, two tank concentrations) was currently out of range. This question was scored on a scale of 0 to 7 by comparing participants' answers with the actual state of the process.

It was very rare for participants to score poorly on this question. The average across all responses was 6.92. Four participants managed a perfect score across 48 administrations of the questionnaire; the lowest individual average was 6.60. There appears to be a correlation between how well people controlled the process and how well they answered this question. However, one should not rush to interpret this as indicating better situational awareness on the part of the people better in control of the process -- rather, if the process was stable, it was easy to answer "nothing is out of range." There also appears to be variation among automation types, and not according to the classical expectation: Manual mode scored lowest at 6.83, with intermediate, adaptable, and automatic nearly tied at 6.94 to 6.95 . It is not clear whether to interpret this as participants being less aware in manual mode, or simply facing a more difficult question in manual mode because people were more likely to have imperfect control of the process in manual mode.

Question 3 on the freeze-probe questionnaire asked participants whether the mixing tank concentrations and outflows had changed, whether any equipment malfunctions had occurred, and whether any safety shutdowns had occurred in the 3 minutes prior to the questionnaire's appearance. The questions was scored on a scale of 0 to 6 by comparing participant's responses with the actual history of the process.

In a few cases, more than one answer was accepted as correct, if more than one interpretation of the process history was possible. If, for instance, an equipment malfunction occurred 4 minutes prior but was not resolved until 2 minutes prior. 
The average score across all responses was 5.75, ranging from 5.27 to 5.96 among participants. Again the people least in control of the process tended to be the people with the lowest score on this question. Interpreting this as a lack of awareness (or, at least, of being overwhelmed by the current demands of the process rather than remembering past events) is clearer here than it was for Question 1.

In contrast to Question 1, the effect of automation type on responses to Question 3 followed the classical pattern of automation level being inversely correlated with automation. The manual mode average was 5.81, intermediate and adaptable were 5.74, automatic 5.73..

2.4.3.2 Learning Effects. There appears to be a learning effect, with the daily averages being 6.86, $6.89,6.93$, and 6.98 on the first through fourth days of the study, respectively.

For Question 3 on the freeze-probe questionnaire, a learning effect also appears to be present (daily averages 5.65, 5.75, 5.77, and 5.84).

\subsubsection{SART}

Participants' responses to several SART items were surprising, showing an unexpected preference for the adaptable mode over all other modes. Participants rated adaptable mode as more stable, ( 3.0 versus 3.6, 3.6, and 3.7) less complex (3.2 versus 3.5, 3.5 and 3.7), less variable (3.5 versus 4.1, 3.8, and 3.9), requiring less division of attention (3.8 versus $3.9,4.3$, and 4.6 ), and leaving more spare mental capacity (5.6 versus 5.5, 5.1 and 4.8 ) than the automatic, intermediate, or manual modes.

They rated automatic mode as most variable of all modes (4.1 versus 3.5, 3.8 and 3.9). This may reflect the fact that participants were trained to vary pump speeds to achieve a steady state, while the automation was willing to repeatedly start and stop pumps and allow concentration in the mixing tanks to oscillate within the acceptable range.

They correctly reported being presented approximately the same quantity of information and being equally familiar with the situation in all 4 modes: all 4 modes do present exactly the same process status information (the intermediate LOA prompts also indicates which parameter is expected to go out of range soonest, when a Intermediate LOA prompt is displayed), and the same events occur in all fourautomation modes. Note that "familiarity with" the situation is not equivalent to ability to cope with the situation, as there are significant differences in performance between modes.

Modest learning effects appeared in the SART responses, with the process being reported as less complex, requiring less concentration, leaving more spare mental capacity, and being more familiar with time.

\subsubsection{Workload}

Participants generally reported low physical demands, but differed widely in their self-assessment of mental and temporal demands and in their reported frustration levels. The two participants who had the greatest difficulty in controlling the process also reported it is more physically demanding than the other participants.

Most participants made an honest effort at self-rating their performance. There is a strong negative correlation between self-reported performance and time out of range and number of safety shutdowns, especially within a single participant's 16 trials.

By contrast, self-reported frustration level was not directly correlated with difficulty maintaining control of the process: some of the highest frustration levels were from participants reporting good performance and relatively low difficulty. The questionnaire cannot distinguish frustration at one's inability to manage the process from being bored by controlling an easy process or being frustrated by quirks of the interface. 
Across all the NASA TLX demand measures, participants reported the highest demands in manual mode, next highest in intermediate, third-highest in adaptable, and lowest in automatic mode. Participants did not self-report any significant differences in performance between modes, despite the objective performance measures indicating worst performance in manual mode.

Learning effects appeared in all of the NASA TLX responses. The strongest of these was a marked decline in frustration level with time. Day-by-day improvements were still occurring after four days.

\section{GENERAL DISCUSSION}

The two extremes of the LOA scale (i.e., manual and fully automatic) produced system performance, fault performance, and SA performance (with one exception) that are consistent with the hypothesized relationships. That is, more automation led to better system performance under normal operating conditions, and poorer SA and system performance under fault conditions. The results for the two middle LOAs are not as straightforward. Consistent with the hypothesis, system performance using the adaptable automation exceeded system performance when using the intermediate LOA. However, contrary to the hypotheses, SA and fault performance were not superior using the adaptable LOA than using the intermediate LOA. The results of this study indicate that although adaptable automation fulfills half if its promise (i.e., it produces system performance closer to fully automatic than intermediate LOAs), it falls short of also enhancing SA and fault performance.

Figure 13 illustrates the overall performance during normal operational changes (as measured by time out of range) and the performance during the fault period. This study has demonstrated the fundamental tradeoff associated with high levels of automation (Onnasch et al., 2013). Automation enhances performance during normal conditions, but increases failure (sometimes catastrophically) of the human-system under the condition of automation failure. In contrast, although manual performance is inferior to automatic performance under normal conditions, it is more stable, and is superior to automatic performance under automation failure conditions. Intermediate automation provided similarly good fault management performance, but did not enhance system performance as well as adaptable automation under normal conditions did.

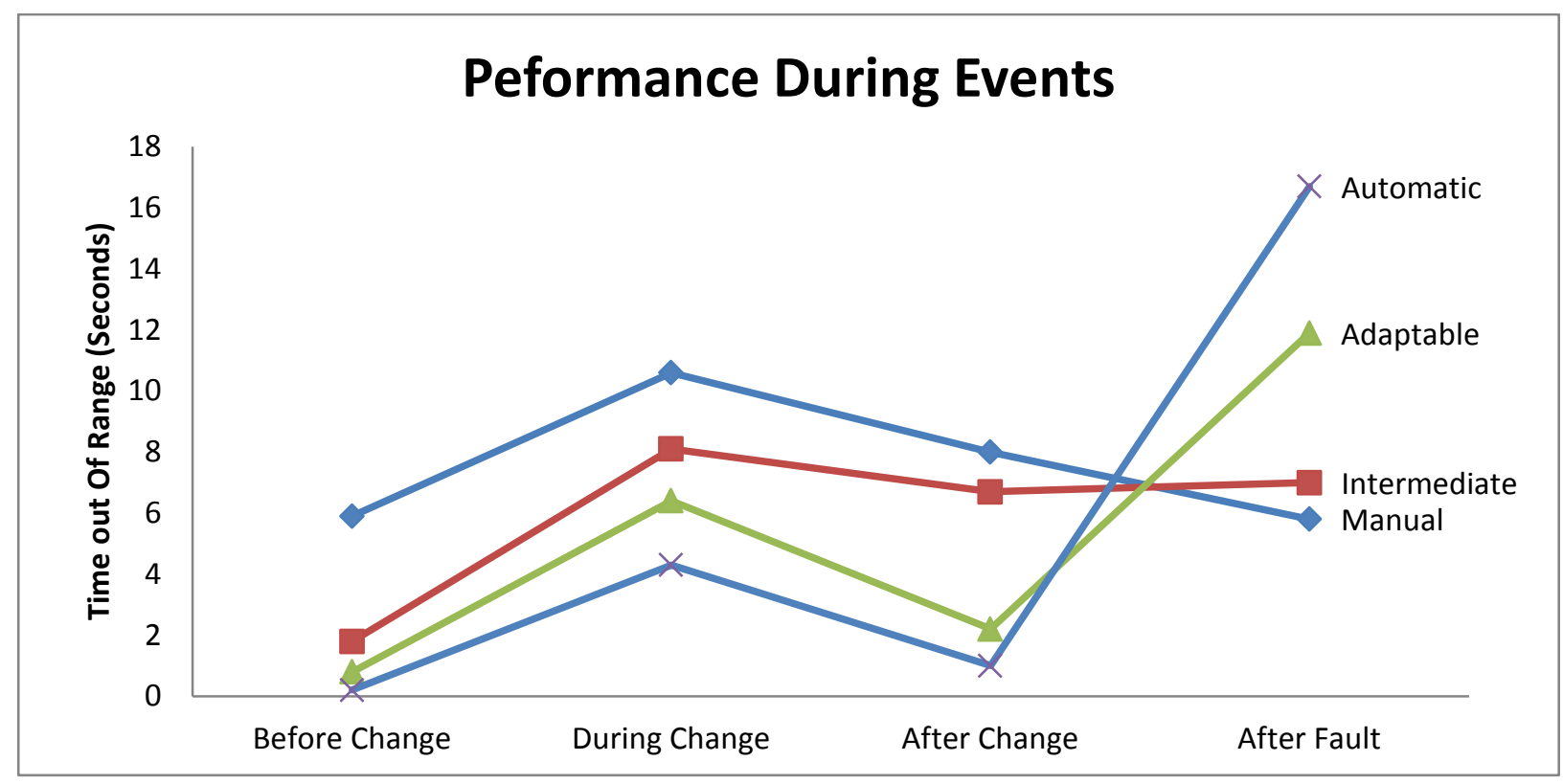

Figure 13. Average time out of range for each event type. Higher scores indicate poorer performance. Please note that the points represent the means of each of the values across all trials. The connecting lines represent change over time for the operational changes (the left three categories of time periods) but the 
faults did not always occur after the operational changes, therefore the line is not meant to represent a consecutive event.

Unfortunately, Experiment Two did not demonstrate that adaptable automation is an effective approach to avoiding that fundamental tradeoff and achieving an optimal combination of system performance, SA and fault management performance. Further research is needed to identify an approach to HAC that will enable higher levels of automation in advanced reactors without succumbing to the fundamental tradeoffs of automation.

\section{FUTURE RESEARCH}

The experimental studies conducted by INL researchers found that there adaptable automation is not an effective way to avoid tradeoffs associated with high levels of automation. However, these studies used simplified process simulations and participants with no or little nuclear operations background. Therefore, it is important to extend the research scope and validity by conducting a series of experiments in a realistic setting using actual nuclear operators. Future research should address both of these gaps by investigating HAC in a realistic and complex context.

The researchers suggest conducting a series of experiments to further investigate the effects of levels of automation on human performance. A part of the experiments should be conducted in the Human Systems Simulations Laboratory, which is a reconfigurable full-scale control room simulator, using control room operators from collaborating utilities (e.g., the Advanced Test Reactor at INL). The other part of the experiments should be conducted in collaboration with industry partners, i.e., utilities and vendors building new plants. When possible, experiments should be conducted at the collaboration partners' control room simulator and with their operators.

The initial studies should compare plant performance, operators' SA, and workload under varying levels of automation (manual, fully automatic, and intermediate levels of automation using different versions of a computerized operator support system . One pilot experimental study should be conducted in the Human Systems Simulations Laboratory to verify the methodology and the selected scenarios. The methodology will be applied to one new large scale reactor design and a SMR design.

Based on the results of these initial studies, researchers will revise the design of the computerized operator support system and the intermediate levels of automation. The modified design will be evaluated in a second round of experimental studies.

The researchers will use the findings from the studies to develop design requirements for human-automation collaboration supporting operator performance in highly automated nuclear power plants, which will be the final product of the research effort.

The results of the work will support design of human-automation interaction in the nuclear industry. Though the results will be directly beneficial to new and advanced reactor designs, the results will also inform the use of advanced technologies that may be installed in existing light water reactors and industries where humans interact with complex and highly automated systems (e.g., the growing hybrid energy industry). Hence, this research is relevant to any facility deploying complex and highly automated systems.

\section{REFERENCES}

Endsley, M.R., and D.B. Kaber (1999). Level of automation effects on performance, situation awareness and workload in a dynamic control task. Ergonomics, Volume 42, pp. 462-492.

Le Blanc, K., and J. Oxstrand (2015). Levels of Automation for Advanced Small Modular Reactors: Impacts on Performance, Workload, and Situation Awareness. Proceedings of the 9th Nuclear 
Plant Instrumentation, Control and Human-Machine Interface Technologies (NPIC\&HMIT) topical meeting of the American Nuclear Society. Charlotte, NC, February 23-26, 2015.

Miller, C. A., and R. Parasuraman (2003). Beyond levels of automation: An architecture for more flexible human-automation collaboration. In Proceedings of the Human Factors and Ergonomics Society Annual Meeting, Volume 47, No. 1, pp. 182-186. SAGE Publications.

Miller, C. A., and R. Parasuraman (2007). Designing for flexible interaction between humans and automation: Delegation interfaces for supervisory control. Human Factors: The Journal of the Human Factors and Ergonomics Society, Volume 49, No. 1, pp. 57-75.

Miller, C., H. Funk, P. Wu, R. Goldman, J. Meisner, and M. Chapman (2005). The Adaptable Approach to Adaptive Automation. In Proceedings of the Human Factors and Ergonomics Society Annual Meeting, Volume 49, No. 1, pp. 15-19). SAGE Publications.

O'Hara, J., J. Higgins, S. Fleger, and V. Barnes (2010). Human-system interfaces to automatic systems: Review guidance and technical basis. Human Factors of advanced reactors (NRC JCN Y-6529), BNL Tech Report No BNL91017-2010, pp. 1-23.

Oxstrand, J., and K. Le Blanc (2014). Effects of Levels of Automation for Advanced Small Modular Reactors: Impacts on Performance, Workload, and Situation Awareness, INL/EXT-14-32639. Idaho National Laboratory. Rev. 0.

Oxstrand, J., J.C. Joe, K.L. Le Blanc, H. Medema, A.M. Whaley, and J. O’Hara (2013a). Framework for Human-Automation Collaboration: Project Status Report, INL/EXT-13-29453. Idaho National Laboratory. Rev. 0.

Oxstrand, J., K.L. Le Blanc, J.C. Joe, A.M. Whaley, H. Medema, and J. O'Hara (2013b). Framework for Human-Automation Collaboration: Conclusions from Four Studies, INL/EXT-13-30570. Idaho National Laboratory. Rev. 0.

Oxstrand, J., J. O’Hara, K.L. Le Blanc, A.M. Whaley, J.C. Joe, and H. Medema (2013c). Development of an Initial Model of Human-Automation Collaboration - Results from a Needs Analysis, INL/EXT-13-28682. Idaho National Laboratory. Rev. 1.

Onnasch, L., C.D. Wickens, H. Li, and D/ Manzey (2013). Human Performance Consequences of Stages and Levels of Automation An Integrated Meta-Analysis. Human Factors: The Journal of the Human Factors and Ergonomics Society, 0018720813501549. 